\title{
Finite-Dimensional Representations of Quantum Affine Algebras
}

\author{
By \\ Tatsuya AKASAKA * and Masaki KASHIWARA *
}

\begin{abstract}
We present a conjecture on the irreducibility of the tensor products of fundamental representations of quantized affine algebras. This conjecture imphes in particular that the irreducibility of the tensor products of fundamental representations is completely described by the poles of $R$-matrices. The conjecture is proved in the cases of type $A_{n}^{(1)}$ and $C_{n}^{(1)}$.
\end{abstract}

\section{Introduction}

In this paper we study finite-dimensional representations of quantum affine algebras. It is known that any finite-dimensional irreducible representation is isomorphic to the irreducible subquotient of a tensor product $\otimes_{\nu} V\left(\pi_{i \nu}\right)_{a_{\nu}}$ containing the highest weight (Drinfeld [7]. Chari-Pressley [2]). Here $V\left(\pi_{1}\right)$ is the fundamental representation corresponding to the fundamental weight $\pi_{\imath}$ and $a_{\nu}$ are spectral parameters. Moreover $\left\{\left(\pi_{i \nu} ; a_{\nu}\right)\right\}_{\nu}$ is uniquely determined up to permutation. This gives a parameterization of the isomorphic classes of finite-dimensional irreducible representations.

However it is not known for example what is the character of those irreducible representations except the complete result for $A_{1}^{(1)}$ ([2]) and some other results due to Chari-Pressley $([2,3,4])$. We have even not known when $\otimes V\left(\pi_{i \nu}\right)_{a_{\nu}}$ itself is irreducible.

In this paper we propose a conjecture on the irreducibility of $\otimes_{\nu} V\left(\pi_{\imath_{\nu}}\right)_{a_{\nu}}$ and prove this conjecture for $A_{n}^{(1)}$ and $C_{n}^{(1)}$.

For $x, y \in \mathbb{C}(q)$, let us denote $x \leq y$ if $x / y$ does not have a pole at $q=0$. We denote by $u_{\imath}$ the highest weight vector of $V\left(\pi_{\imath}\right)$.

Received April 24, 1997. Revised August 18, 1997.

1991 Mathematics Subject Classification (́s) :17B37

* Research Institute for Mathematical Sciences. Kyoto University, Kyoto 606-01, Japan

The first author is supported by the JSPS Research Fellowship for Young Scientists. 
Comjecture $\mathbb{1}$.

(1) If $a_{1} \leq \cdots \leq a_{N}$, then $V\left(\pi_{i_{1}}\right)_{a_{1}} \otimes \cdots \otimes V\left(\pi_{i_{N}}\right)_{a_{N}}$ is generated by $u_{i_{1}} \otimes \cdots \otimes u_{i_{N}}$ as a $U_{q}^{\prime}(\mathfrak{g})$-module.

(2) If $a_{1} \geq \cdots \geq a_{N}$, then any non-zero $U_{q}^{\prime}(\mathfrak{g})$-submodule of $V\left(\pi_{t_{1}}\right){ }_{a_{1}} \otimes \cdots \otimes$ $V\left(\pi_{t_{N}}\right)_{a_{N}}$ contains $u_{{ }_{11}} \otimes \cdots \otimes u_{i_{N}}$.

Here $U_{q}^{\prime}(\mathrm{g})$ is the quantum affine algebra without derivation (see $§ 1.1$ ).

This conjecture implies in particular the following consequences.

Claim 1 . If $a_{1} \leq a_{2}$, then the normalized $R$-matrix

$$
R_{i, j}^{\text {nor }}(x, y): V\left(\pi_{i}\right)_{x} \otimes V\left(\pi_{j}\right)_{y} \rightarrow V\left(\pi_{j}\right)_{y} \otimes V\left(\pi_{\imath}\right)_{x}
$$

does not have a pole at $(x, y)=\left(a_{1}, a_{2}\right)$.

Here $R_{i, j}^{\text {nor }}(x, y)$ is so normalized that it sends $u_{t} \otimes u_{\jmath}$ to $u_{j} \otimes u_{t}$.

Claim 2. $V\left(\pi_{t_{1}}\right)_{a_{1}} \otimes \cdots \otimes V\left(\pi_{i_{N}}\right)_{a_{N}}$ is irreducible if and only if the R-matrix

$$
R_{i \nu, l_{\mu}}^{\text {nor }}(x, y): V\left(\pi_{i \nu}\right)_{x} \otimes V\left(\pi_{\imath \mu}\right)_{y} \rightarrow V\left(\pi_{\imath \mu}\right)_{y} \otimes V\left(\pi_{\imath \nu}\right)_{x}
$$

does not have a pole at $(x, y)=\left(a_{\nu}, a_{\mu}\right)$ for any $1 \leq \nu, \mu \leq N(\nu \neq \mu)$.

Claim 3. Assume that $R_{l \nu, \imath u}^{\text {nor }}(x, y)$ has no pole at $(x, y)=\left(a_{\nu}, a_{\mu}\right)$ for any 1 $\leq \mu<\nu \leq N$. Then the submodule generated by $u_{i_{1}} \otimes \cdots \otimes u_{u_{N}}$ is an irreducible submodule of $V\left(\pi_{11}\right)_{a_{\nu}} \otimes \cdots \otimes V\left(\pi_{i_{N}}\right)_{a_{N}}$. Conversely, any finite-dimensional irreducible integrable module is obtained in this way.

Claim 4. If $M$ and $M^{\prime}$ are irreducible finite-dimensional integrable $U_{q}^{\prime}(\mathrm{g})$ modules, then $M \otimes M_{z}^{\prime}$ is an irreducible $U_{q}^{\prime}(\mathrm{g})$-module except for finitely many' $z$.

The plan of the paper is as follows In $\S 1$. we fix notations and explain the results used after. We announce non published results but they can be directly checked for the $A_{n}^{(1)}$ and $C_{n}^{(1)}$ cases. In $\S 2$, we announce the main conjecture and discuss its consequences. In $\S 3$, we reduce the main conjecture to another auxiliary conjecture, which will be proved in the case $A_{n}^{(1)}$ and $C_{n}^{(1)}$ in $\S 4$. In the appendix. we shall calculate the explicit form of the normalized $R$-matrices and the universal $R$-matrices between fundamental representations of $A_{n}^{(1)}$ and $C_{n}^{(1)}$.

The authors are grateful to K. Takemura for his helpful comments on this work.

\section{Notations}

1.1. Qunantized affine ælgebras. Let $\left(a_{\imath \jmath}\right)_{\imath, j \in I}$ be a generalized Cartan matrix of affine type. We choose a $\mathbb{Q}$-vector space $\mathrm{t}$ of dimension $I+1$ and simple roots $\alpha_{1} \in \mathrm{t}^{*}$ and simple coroots $h_{\imath} \in \mathrm{t}$ such that $\left\langle h_{\imath}, \alpha_{j}\right\rangle=a_{\imath \jmath}$. We assume further that $\alpha_{\imath}$ and $h_{\imath}$ are linearly independent. Set $Q=\sum_{\imath} \mathbb{Z} \alpha_{\imath}$ and $Q^{\vee}=\sum_{i} \mathbb{Z} h_{\imath}$. 
Let $\delta=\sum a_{i} \alpha_{i}$ be the smallest positive imaginary root and let $c=\sum a_{i}^{\vee} h_{i} \in Q^{\vee}$ be the center. Set $\mathrm{t}_{\mathrm{cl}}^{*}=\mathrm{t}^{*} / \mathrm{Q} \delta$ and let $\mathrm{cl}: \mathrm{t}^{*} \rightarrow \mathrm{t}_{\mathrm{cl}}^{*}$ be the projection. We set $\mathrm{t}^{* 0}=\{\lambda \in$ $\left.\mathfrak{t}^{*} ;\langle c, \lambda\rangle=0\right\}$ and $\mathfrak{t}_{\mathrm{cl}}^{* 0}=\mathrm{cl}\left(\mathrm{t}^{* 0}\right)$.

We take a non-degenerate symmetric bilinear form $(\cdot$,$) on t^{*}$ such that

$$
\left\langle h_{i}, \lambda\right\rangle=\frac{2\left(\alpha_{i}, \lambda\right)}{\left(\alpha_{i}, \alpha_{\imath}\right)} \text { for any } i \in I \text { and } \lambda \in \mathrm{t}^{*} \text {. }
$$

We normalize it by

$$
\langle c, \lambda\rangle=(\delta, \lambda) \text { for any } \lambda \in \mathrm{t}^{*}
$$

We identify sometimes $t$ and $t^{*}$ by this symmetric form.

Let us take a (weight) lattice $P \subset \mathrm{t}^{*}$ such that $\alpha_{\imath} \in P$ and $h_{i} \in P^{*}$ for every $i$ $\in I$. We assume further that $P$ contains $\Lambda_{\imath}$ satisfying $\left\langle h_{j}, \Lambda_{\imath}\right\rangle=\delta_{\imath j}$ and that $P \cap$ $\mathbb{Q} \delta=\mathbb{Z} \delta$. We set $P_{\mathrm{cl}}=P / \mathbb{Z} \delta \subset \mathrm{t}_{\mathrm{cl}}^{*}, P^{0}=\{\lambda \in P ;\langle c, \lambda\rangle=0\} \subset \mathrm{t}^{* 0}$, and $P_{\mathrm{cl}}^{0}=\mathrm{cl}\left(P^{0}\right) \subset \mathrm{t}_{\mathrm{cl}}^{* 0}$. Note that the dual lattice of $Q^{\vee}$ coincides with $P_{\mathrm{cl}} \cong \bigoplus_{t \in I} \mathbb{Z} \mathrm{Cl}\left(\Lambda_{i}\right)$.

Let $\gamma$ be the smallest positive integer such that

$$
\gamma\left(\alpha_{i}, \alpha_{\imath}\right) / 2 \in \mathbb{Z} \text { for any } i \in I .
$$

Then the quantized affine algebra $U_{q}(\mathfrak{g})$ is the algebra over $k=\mathbf{Q}\left(q^{1 / \gamma}\right)$ generated by the symbols $e_{i}, f_{i}(i \in I)$ and $q(h)\left(h \in \gamma^{-1} P^{*}\right)$ satisfying the following defining relations.

(1) $q(h)=1$ for $h=0$.

(2) $q\left(h_{1}\right) q\left(h_{2}\right)=q\left(h_{1}+h_{2}\right)$ for $h_{1}, h_{2} \in \gamma^{-1} P^{*}$.

(3) For any $i \in I$ and $h \in \gamma^{-1} P^{*}$,

$$
\begin{aligned}
& q(h) e_{i} q(h)^{-1}=q^{\left\langle h, \alpha_{\imath}\right\rangle} e_{\imath} \quad \text { and } \\
& q(h) f_{\imath} q(h)^{-1}=q^{-\left\langle h, \alpha_{t}\right\rangle} f_{\imath} .
\end{aligned}
$$

(4) $\left[e_{\imath}, f_{j}\right]=\delta_{\imath l} \frac{t_{i}-t_{i}^{-1}}{q_{\imath}-q_{\imath}^{-1}}$ for $i, j \in I$. Here $q_{i}=q^{\left(\alpha_{t}, \alpha_{i}\right) / 2}$ and $t_{i}=q\left(\frac{\left(\alpha_{i}, \alpha_{i}\right)}{2} h_{i}\right)$.

(5) (Serre relations) For $i \neq j$,

$$
\sum_{k=0}^{b}(-1)^{k} e_{i}^{(k)} e_{j} e_{i}^{(b-k)}=\sum_{k=0}^{b}(-1)^{k} f_{i}^{(k)} f_{j} f_{i}^{(b-k)}=0 .
$$

Here $b=1-\left\langle h_{i}, \alpha_{j}\right\rangle$ and

$$
\begin{array}{ll}
e_{i}^{(k)}=e_{i}^{k} /[k]_{i} !, & f_{i}^{(k)}=f_{i}^{k} /[k]_{i} !, \\
{[k]_{i}=\left(q_{i}^{k}-q_{\imath}^{-k}\right) /\left(q_{i}-q_{\imath}^{-1}\right),} & {[k]_{i} !=[1]_{i} \cdots[k]_{i} .}
\end{array}
$$

We denote by $U_{q}^{\prime}(\mathfrak{g})$ the subalgebra of $U_{q}(\mathfrak{g})$ generated by $\ell_{\imath}, f_{\imath}(i \in I)$ and $q(h)\left(h \in \gamma^{-1} Q^{\vee}\right)$. 
In this paper we consider only $U_{q}^{\prime}(\mathfrak{g})$. A $U_{q}^{\prime}(\mathfrak{g})$-module $M$ is called integrable if $M$ has the weight decomposition $M=\bigoplus_{\lambda \in P_{\mathrm{c} 1}} M_{\lambda}$ where $M_{\lambda}=\{u \in M ; q$ (h) $\left.u=q^{\langle h, \lambda\rangle} u\right\}$, and if $M$ is $U_{q}(\mathrm{~g})_{i}$-locally finite (i.e. $\operatorname{dim} U_{q}(\mathrm{~g})_{\imath} u<\infty$ for every $u \in M)$ for every $i \in I$. Here $U_{q}(\mathrm{~g})_{i}$ is the subalgebra generated by $e_{i}, f_{i}$ and $t_{i}$.

We use the coproduct $\Delta$ of $U_{q}(\mathrm{~g})$ given by

$$
\begin{aligned}
\Delta(q(h)) & =q(h) \otimes q(h), \\
\Delta\left(e_{i}\right) & =e_{i} \otimes t_{i}^{-1}+1 \otimes e_{i}, \\
\Delta\left(f_{l}\right) & =f_{i} \otimes 1+t_{i} \otimes f_{i},
\end{aligned}
$$

so that the lower crystal bases behave well under the corresponding tensor products $([12])$.

1.2. Finite-dimensional representations. Let $W \subset$ Aut $\left(t^{*}\right)$ be the Weyl group, and let $l: W \rightarrow \mathbb{Z}$ be the length function. Since $\delta$ is invariant by $W$, we have the group homomorphism $\mathrm{cl}_{0}: W \rightarrow$ Aut $\left(\mathfrak{t}_{\mathrm{cl}}^{* 0}\right)$. Let $W_{\mathrm{cl}} \subset$ Aut $\left(\mathfrak{t}_{\mathrm{cl}}^{* 0}\right)$ be the image of $W$ by $\mathrm{cl}_{0}$. Then $W_{\mathrm{cl}}$ is a finite group. Let us take $i_{0} \in I$ such that $W_{\mathrm{cl}}$ is generated by $\operatorname{cl}_{0}\left(s_{\imath}\right)\left(i \in I_{0}=I \backslash\left\{i_{0}\right\}\right)$ and that $a_{20}^{\vee}=1$. Such an $i_{0}$ is unique up to Dynkin diagram automorphism. Hereafter we write 0 instead of $i_{0}$.

Let us denote by $W_{0}$ the subgroup of $W$ generated by $s_{\imath}\left(i \in I_{0}=I \backslash\{0\}\right)$. Then $W_{0}$ is isomorphic to $W_{\mathrm{cl}}$. The kernel of $W \rightarrow W_{\mathrm{cl}}$ is the commutative group $\left\{t(\xi) ; \xi \in Q_{\mathrm{cl}} \cap Q_{\mathrm{cl}}^{\vee}\right\}$. Here $Q_{\mathrm{cl}}=\operatorname{cl}(Q)=\sum_{t \in I} \mathbb{Z} \mathrm{cl}\left(\alpha_{\imath}\right)$ and $Q_{\mathrm{cl}}^{\vee}=\operatorname{cl}\left(Q^{\vee}\right)=$ $\sum_{t \in I_{0}} \mathbb{Z} \mathrm{cl}\left(h_{\imath}\right)$ and $t(\xi)$ is the automorphism of $\mathrm{t}^{*}$ given by

$$
t(\xi)(\lambda)=\lambda+(\delta, \lambda) \xi^{\prime}-\left(\xi^{\prime}, \lambda\right) \delta-\frac{\left(\xi^{\prime}, \xi^{\prime}\right)}{2}(\delta, \lambda) \delta
$$

for $\xi^{\prime} \in \mathrm{t}^{*}$ such that $\mathrm{cl}\left(\xi^{\prime}\right)=\xi$.

The following lemma is well-known.

Lemma 1.1. Let $\xi \in Q_{\mathrm{c} 1} \cap Q_{\mathrm{cl}}^{\vee}$ and $w \in W_{0}$.

(i) If $\xi$ is dominant (with respect to $I_{0}$ ), then we have

$$
l(w \circ t(\xi))=l(w)+l(t(\xi)) .
$$

(ii) If $\xi$ is regular and dominant, then we have

$$
l(t(\xi) \circ w)=l(t(\xi))-l(w) .
$$

Let us choose $i_{1}$ such that $W_{\mathrm{cl}}$ is generated by $\mathrm{cl}_{0}\left(s_{i}\right)\left(i \in I \backslash\left\{i_{1}\right\}\right)$ and that $a_{i_{1}}$ $=1$. For any $z \in k \backslash\{0\}$, let $\phi(z)$ be the automorphism of $U_{q}^{\prime}(\mathrm{g})$ given by

$$
\begin{aligned}
\phi(z)\left(e_{\imath}\right) & =z^{\delta_{t,+}} e_{\imath}, \\
\phi(z)\left(f_{i}\right) & =z^{-\delta_{t} \mathrm{nt}} f_{\imath}, \\
\phi(z)(q(h)) & =q(h) .
\end{aligned}
$$


For a $U_{q}^{\prime}(\mathfrak{g})$-module $M$, let $M_{z}$ be the $U_{q}^{\prime}(\mathfrak{g})$-module with $M$ as its underlying $k$-vector space and with $U_{q}^{\prime}(\mathfrak{g}) \stackrel{\psi(z)}{\longrightarrow} U_{q}^{\prime}(\mathfrak{g}) \rightarrow$ End $(M)$ as the action of $U_{q}^{\prime}(\mathfrak{g})$. Then $M \mapsto M_{z}$ is a functor satisfying $(M \otimes N)_{z} \cong M_{z} \otimes N_{z}$. This definition extends to the case $z \in K \backslash\{0\}$ for a field extension $K \supset k$.

If $M$ is a finite-dimensional integrable $U_{q}^{\prime}(\mathfrak{g})$-module, then the weights of $M$ are contained in $P_{\mathrm{cl}}^{0}$.

1.3. Fundamental representations. We set $\pi_{i}=\operatorname{cl}\left(\Lambda_{i}-a_{i}^{\vee} \Lambda_{0}\right)$ for $i \in I_{0}$. Then $\left(\pi_{\imath}\right)_{\imath \in I_{0}}$ forms a basis of $P_{\mathrm{cl}}^{0}$. We call $\pi_{\imath}$ a fundamental weight (of level 0 ).

For $i \in I_{0}$, there exists an irreducible integrable $U_{q}^{\prime}(\mathrm{g})$-module $V\left(\pi_{i}\right)$ satisfying the following properties.

(1) The weights of $V\left(\pi_{i}\right)$ are contained in the convex hull of $W_{\mathrm{cl}} \pi_{t}$.

(2) $\operatorname{dim} V\left(\pi_{2}\right)_{\pi_{t}}=1$.

(3) For any $\mu \in W_{\mathrm{cl}} \pi_{i} \subset P_{\mathrm{cl}}^{0}$, we can associate a non-zero vector $\iota_{\mu}$ of weight $\mu$ such that

$$
u_{s, \mu}= \begin{cases}f_{j}^{\langle\langle h, \mu\rangle)} u_{\mu} & \text { if }\left\langle h_{,}, \mu\right\rangle \geq 0, \\ e_{j}^{(-\langle h, \mu \nu)} u_{\mu} & \text { if }\left\langle h_{\jmath}, \mu\right\rangle \leq 0\end{cases}
$$

for any $j \in I$.

Then $V\left(\pi_{2}\right)$ is unique up to an isomorphism. Moreover $V\left(\pi_{\imath}\right)$ has a global crystal base. We call $V\left(\pi_{\imath}\right)$ a fundamental representation. Then $V\left(\pi_{\imath}\right)$ has a non-degenerate symmetric bilinear form $(\bullet,)^{\prime}$ such that ${ }^{t} e_{\imath}=f_{l}$ and ${ }^{t} q(h)=q(h)$. Hence the duality is given as follows. Let $w_{0}$ be the longest element of $W_{0}$. Then for $i \in I_{0}$ there exists $i^{*} \in I_{0}$ such that

$$
\pi_{\imath *}=-w_{0} \pi_{i}
$$

(Remark that $i \mapsto i^{*}$ with $0^{*}=0$ gives a Dynkin diagram automorphism.) Then the right dual of $V\left(\pi_{1}\right)$ is $V\left(\pi_{1 *}\right)_{p *}$ with the duality morphisms:

$$
k \rightarrow V\left(\pi_{i}\right)_{p *} \otimes V\left(\pi_{\imath}\right) \text { and } V\left(\pi_{\imath}\right) \otimes V\left(\pi_{\imath}\right)_{p *} \rightarrow k
$$

with $p^{*}=(-1)^{\left\langle\rho^{\vee}, \delta\right\rangle} q^{(\rho, \delta)}$. Here $\rho$ and $\rho^{\vee}$ are defined by: $\left\langle h_{i}, \rho\right\rangle=1$ and $\left\langle\rho^{\vee}, \alpha_{i}\right\rangle=$ 1 for every $i \in I$. Usually $(\rho, \delta)=\sum_{i \in I} a_{\imath}^{\vee}$ is called the dual Coxeter number and $\left\langle\rho^{\vee}, \delta\right\rangle=\sum_{t \in I} a_{\imath}$ the Coxeter number.

Let $m_{\imath}$ be a positive integer such that

$$
W\left(\Lambda_{\imath}-a_{\imath}^{\vee} \Lambda_{0}\right)=\left(\Lambda_{\imath}-a_{\imath}^{\vee} \Lambda_{0}\right)+\mathbf{Z} m_{\imath} \delta .
$$

We have $m_{i}=\left(\alpha_{i}, \alpha_{i}\right) / 2$ in the case where $g$ is the dual of an untwisted affine algebra, and $m_{\imath}=1$ in the other cases.

Then for $z, z^{\prime} \in K^{*}$, we have 


$$
V\left(\pi_{i}\right)_{z} \cong V\left(\pi_{\imath}\right)_{z^{\prime}} \text { if and only if } z^{m_{i}}=z^{m_{i}} .
$$

Hence we set

$$
V\left(\pi_{i} ; z^{m_{i}}\right)=V\left(\pi_{i}\right)_{z}
$$

The following theorem is announced by Drinfeld ([7]) in Yangian case, and its proof is given by Chari-Pressley $([3,4])$.

Theorem $\mathbb{1}_{0}$. Let $K \supset k$ be an algebraically closed field and let $M$ be an irreducible finite-dimensional $U_{q}^{\prime}(\mathfrak{g})_{K}$-module. Then there exist $i_{1}, \ldots, i_{N} \in I_{0}$ and $z_{1}, \ldots, z_{N} \in K \backslash\{0\}$ such that $M$ is isomorphic to a unique irreducibe subquotient of $V\left(\pi_{i_{1}} ; z_{1}\right) \otimes \cdots \otimes V\left(\pi_{i_{N}} ; z_{N}\right)$ containing the weight $\sum_{\nu=1}^{N} \pi_{i_{\nu}}$. Moreover, $\left\{\left(i_{1} ; z_{1}\right) \ldots .\right.$, $\left.\left(i_{N} ; z_{N}\right)\right\}$ is unique up to permutations.

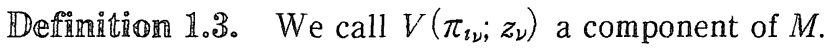

1.4. Extremal vectors. We say that a crystal $B$ over $U_{q}^{\prime}(\mathrm{g})$ is a regular crystal if, for any $\underset{\neq}{\subset \underset{c}{\subset} I, B}$ is isomorphic to the crystal associated with an integrable $U_{q}\left(\mathfrak{g}_{J}\right)$-module. Here $U_{q}\left(\mathfrak{g}_{J}\right)$ is the subalgebra of $L_{q}^{\prime}(\mathfrak{g})$ generated by $e_{i}, f_{\imath}$ and $t_{i}(i \in J)$. This condition is equivalent to saying that the same assertion holds for any $J \subset I$ with two elements (see [15, Proposition 2.4.4]).

By [14], the Weyl group $W$ acts on any regular crystal. This action $S$ is given by

$$
S_{S} b= \begin{cases}\widetilde{f}_{i}^{\left\langle h_{t}, W t(b)\right\rangle} b & \text { if }\left\langle h_{i}, \text { wt }(b)\right\rangle \geq 0 \\ \tilde{e}_{\imath}^{-\left\langle h_{t}, W t(b)\right\rangle} b & \text { if }\left\langle h_{\imath}, \text { wt }(b)\right\rangle \leq 0 .\end{cases}
$$

A vector $b$ of a regular crystal $B$ is called $i$-extremal if $\tilde{e}_{i} b=0$ or $\tilde{f}_{i} b=0$. We call $b$ an extremal vector if $S_{w} b$ is $i$-extremal for any $w \in W$ and $i \in I$.

Lemma 1.4. For any $\lambda, \mu \in \mathrm{t}_{\mathrm{cl}}^{* 0}$ in the same $W_{\mathrm{cl}}$-orbit, we can find $i_{1}, \ldots, i_{N} \in I$ such that

$$
\begin{aligned}
& \mu=s_{i_{N}} \cdots s_{i_{1}} \lambda, \\
& \left\langle h_{i_{k}}, s_{i_{k-1}} \cdots s_{i_{1}} \lambda\right\rangle>0 \text { for any } 1 \leq k \leq N .
\end{aligned}
$$

Proof. It is enough to prove the statement above for a regular integral anti-dominant (with respect to $I_{0}$ ) weight $\lambda$ and the dominant weight $\mu \in W \lambda$. We may assume further $\lambda \in Q_{\mathrm{cl}} \cap Q_{\mathrm{cl}}^{v}$. Let $w_{0}$ be the longest element of $W_{0}$. By Lemma 1.1, we have

$$
\begin{aligned}
l(t(\lambda)) & =l(t(-\lambda)) \\
& =l\left(w_{0}\right)+l\left(t(-\lambda) w_{0}\right) \\
& =l\left(w_{0}\right)+l\left(w_{0} t(\lambda)\right) .
\end{aligned}
$$


Take a reduced expression $w_{0} t(\lambda)=s_{i_{N}} \cdots s_{i_{1}}$. Then for $1 \leq k \leq N$ we have $l(t(\lambda)$ $\left.s_{\imath_{1}} \cdots s_{t_{k}}\right)=l(t(\lambda))-k$ and hence $t(\lambda) s_{i_{1}} \cdots s_{t_{k-1}} \alpha_{k}$ is a negative root. Since it is equal to $s_{t_{1}} \cdots s_{i_{k-1}} \alpha_{k}-\left(\lambda, s_{t_{1}} \cdots s_{t_{k-1}} \alpha_{k}\right) \delta$ and $s_{t_{1}} \cdots_{t_{k-1}} \alpha_{k}$ is a positive root, we conclude

$$
\left(\lambda, s_{i 1} \cdots s_{t_{k-1}} \alpha_{k}\right)>0 \text {. }
$$

On the other hand we have the equality $s_{l_{N}} \cdots s_{l_{1}} \lambda=w_{0} t(\lambda) \lambda=w_{0} \lambda$ in $\mathrm{t}_{\mathrm{cl}}^{* 0}$. Hence it is equal to $\mu$.

For a regular crystal $B, b \in B$ and $i \in I$, let us denote by $\tilde{e}_{i}^{\max } b$ the $i$-highest weight vector in the $i$-string containing $i$. Namely we have

$$
\tilde{e}_{i}^{\max } b=\tilde{e}_{i}^{\varepsilon_{i}(b)} b .
$$

Lemma 1.5. Let $B$ be a finite regular crystal with level 0 (with weight in $\left.P_{\mathrm{cl}}^{0}\right)$.

(1) For $b \in B$, there arc $i_{1, \ldots, i_{N}} \in I$ such that $\widetilde{e}_{i_{N}}^{\max } \ldots \tilde{e}_{i_{1}}^{\max } b$ is an extramal vector.

(2) Any vector in the $W$-orbit of an extremal vector $b$ of $B$ is written in the form $\tilde{e}_{i_{N}}^{\max } \ldots \tilde{e}_{i_{1}}^{\max } b$.

Proof. Let us set $F_{l}=\left\{\widetilde{e}^{\max } \ldots \widetilde{e}_{i_{1}}^{\max } b ; i_{1}, \ldots, i_{l} \in I\right\}, F=\cup_{l \geq 0} F_{l}$. Replacing $b$ with $b^{\prime} \in F$ with maximal $\left(\mathrm{wt}\left(b^{\prime}\right)\right.$, wt $\left.\left(b^{\prime}\right)\right)$, we may assume from the beginning that $\left(\mathrm{wt}\left(b^{\prime}\right)\right.$, wt $\left.\left(b^{\prime}\right)\right) \leq(\mathrm{wt}(b)$. wt $(b))$ for any $b^{\prime} \in F$. Since $\left(\operatorname{wt}\left(b^{\prime}\right)\right.$, wt $\left.\left(b^{\prime}\right)\right) \geq$ (wt $(b)$, wt $(b))$, we have $\left(\operatorname{wt}\left(b^{\prime}\right), \operatorname{wt}\left(b^{\prime}\right)\right)=(\operatorname{wt}(b)$, wt $(b))$ for any $b^{\prime} \in F$, and hence any $b^{\prime} \in F$ is $i$-extremal for every $i \in I$. Moreover the weight of $b^{\prime}$ is in the $W_{\mathrm{cl}}{ }^{-}$orbit of wt $(b)$. Then for any weight $\mu$ of $F$ and $i$ such that $\left\langle h_{\imath}, \mu\right\rangle \leq 0, S_{s_{t}}$ sends injectively $F_{\mu}$ to $F_{s_{\mu} \mu}$. Hence \#( $\left.F_{\mu}\right) \leq \#\left(F_{s_{\mu}}\right)$, and Lemma 1.4 asserts that they must be equal. Therefore $S_{i}: F_{\mu} \rightarrow F_{s i \mu}$ is bijective. This shows that $F$ is stable by all $S_{s_{t}}$. Thus we have (1) and (2).

Lemma 1.6. Let $B_{1}$ and $B_{2}$ be two finite regular crystals. Let $b_{1}$ and $b_{2}$ be vectors in $B_{1}$ and $B_{2}$, respectively.

(1) If $b_{1}$ and $b_{2}$ are extremal vectors and if their weights are in the same Weyl chamber, then $b_{1} \otimes b_{2}$ is extremal.

(2) Conversely if $b_{1} \otimes b_{2}$ is extremal, then $b_{1}$ and $b_{2}$ are extremal vectors and their weights are in the same Weyl chamber.

Proof. (1) is obvious because $S_{w}\left(b_{1} \otimes b_{2}\right)=S_{w} b_{1} \otimes S_{w} b_{2}$ under this condition.

We shall prove (2). Since $\widetilde{e}_{i_{1}}^{\max } \cdots \widetilde{e}_{i_{N}}^{\max }\left(b_{1} \otimes b_{2}\right)=\tilde{e}_{i_{1}}^{\max } \cdots \widetilde{e}_{i_{N}}^{\max } b_{1} \otimes b_{2}^{\prime}$ for some $b_{2}^{\prime} \in B_{2}$, the preceding lemma implies that $b_{1}$ is extremal. Similarly $b_{2}$ is extremal. It remains to prove that wt $\left(b_{1}\right)$ and $\mathrm{wt}\left(b_{2}\right)$ are in the same Weyl chamber. Let us show first that wt $\left(b_{1} \otimes b_{2}\right)$ and $\mathrm{wt}\left(b_{1}\right)$ are in the same Weyl chamber. We may assume without loss of generality that wt $\left(b_{1} \otimes b_{2}\right)$ is dominant (with 
respect to $\left.I_{0}\right)$. Then $\widetilde{e_{i}}\left(b_{1} \otimes b_{2}\right)=0$ for every $i \in I_{0}$. Hence $\tilde{e_{2}} b_{1}=0$. Hence wt $\left(b_{1}\right)$ is dominant. Hence wt $\left(b_{1} \otimes b_{2}\right)$ and wt $\left(b_{1}\right)$ are in the same Weyl chamber. Similarly wt $\left(b_{1} \otimes b_{2}\right)$ and wt $\left(b_{2}\right)$ are in the same Weyl chamber. Thus wt $\left(b_{1}\right)$ and wt $\left(b_{2}\right)$ are in the same Weyl chamber.

Definition 1.7. We say that a finite regular crystal $B$ is simple if $B$ satisfies

(1) There exists $\lambda \in P_{\mathrm{cl}}^{0}$ such that the weights of $B$ are in the convex hull of $W_{\mathrm{cl}} \lambda$.

(2) 菑 $\left(B_{\lambda}\right)=1$.

(3) The weight of any extremal vector is in $W_{\mathrm{cl}} \lambda$.

Prposition 1.8. The crystal graph of the fundamental representations is simple.

The proof will be given elsewhere. However we can easily check this for the $A_{n}^{(1)}$ and $C_{n}^{(1)}$ cases.

Lemma 1.9. A simple crystal $B$ is connected.

Proof. In fact, any vector is connected with an extremal vector by Lemma 1.5.

$\mathbb{L}$ emma $\mathbb{1}$.10. The tensor product of simple crystals is also simple.

Proof. This immediately follows from Lemma 1.6.

Proposition 1.11 . Let $M$ be a finite-dimensional integrable $L_{q}^{\prime}(\mathrm{g})$-inodule with a crystal base $(L, B)$. Assume the folllwing conditions.

(1.8) $B$ is connected.

(1.9) There exists a weight $\lambda \in P_{\mathrm{cl}}^{0}$ such that $\operatorname{dim}\left(M_{\lambda}\right)=1$.

Then $M$ is irreducible.

Proof. We shall show first that $M_{\lambda}$ generates $M$. Set $N=U_{q}^{\prime}(\mathfrak{g}) M_{\lambda}$ and $\bar{N}=$ $(L \cap N) /(q L \cap N) \subset L / q L$. Then $\bar{N}$ is invariant by $\widetilde{e_{\imath}}$ and $\tilde{f}_{l}$. Hence $\bar{N}$ contains $B$, and Nakayama's lemma asserts that $N=M$. By duality, any non-zero submodule of $M$ contains $M_{\lambda}$. Therefore $M$ is irreducible.

Corollary 1.12. A finite-dimensional $U_{q}^{\prime}(\mathfrak{g})$-module with a simple crystal base is irreducible.

Corollary 1.13 . For $i_{1}, \ldots, i_{N} \in I_{0}, V\left(\pi_{\imath_{1}}\right) \otimes \cdots \otimes V\left(\pi_{i_{N}}\right)$ is irreducible.

We define similarly an extremal vector of an integrable $U_{q}^{\prime}(\mathrm{g})$-module.

Definition 1.14. Let $v$ be a weight vector of an integrable $U_{q}^{\prime}(\mathfrak{g})$-module. We call $v^{\prime}$ extremal if the weights of $U_{q}^{\prime}(\mathfrak{g}) v$ are contained in the convex hull of $W$ wt $\left(v^{\prime}\right)$. 
When the weight of $v$ is of level 0 and dominant (with respect to $I_{0}$ ), $v$ is extremal if and only if wt $\left(U_{q}^{\prime}(\mathrm{g}) v\right) \subset \mathrm{wt}(v)+\sum_{t \in I_{0}} \mathbb{Z}_{\leq 0} \mathrm{cl}\left(\alpha_{i}\right)$. In this case, we call $v$ a dominant extemal vector.

Since the following proposition is not used in this paper, the proof will be given elsewhere.

Proposition 1.15. Let $v$ be a weight vector of an integrable $U_{q}^{\prime}(\mathrm{g})$-module. The following two conditions are equivalent.

(1) $v$ is an extremal vector.

(2) We can associate a vector $v_{w}$ of weight $w \mathrm{wt}(v)$ to each $w \in W$ satisfying the following properties:

(a) $v_{w}=v$ if $w=e$,

(b) If $i \in I$ and $w \in W$ satisfy $\left\langle h_{\imath}, w\right.$ wt $\left.(v)\right\rangle \geq 0$, then $e_{\imath} v_{w}=0$ and $v_{s_{t} w}=$ $f_{i}^{\left(\left\langle h_{t}, w \mathrm{wt}(v)\right\rangle\right)} v^{\prime} w$,

(c) If $i \in I$ and $w \in W$ satisfy $\left\langle h_{\imath}, w \mathrm{wt}(v)\right\rangle \leq 0$, then $f_{i} v_{w}=0$ and $v_{s_{s} w}=$ $e_{i}^{\left\{-\left\langle h_{w}, u w \mathrm{wt}(w)\right\rangle\right.} v_{w}$.

The implication $(1) \Rightarrow(2)$ is obvious. I).

Let us denote by $U_{q}^{\prime}(\mathfrak{b})$ the subalgebra of $U_{q}^{\prime}(\mathfrak{g})$ generated by $t_{i}$, and $e_{\imath}(i \in$

Proposition 1.16. Let $M$ be a finite-dimensional integrable $U_{q}^{\prime}(\mathfrak{g})$-module. Then any $U_{q}^{\prime}(\mathfrak{b})$-submodule of $M$ is a $U_{q}^{\prime}(\mathfrak{g})$-submodule.

Proof. Let $N$ be a $U_{q}^{\prime}(\mathfrak{b})$-submodule. For any pair of weights $\lambda$ and $\mu$ conjugate by $W_{\mathrm{cl}}$, there exist $i_{1}, \ldots, i_{l}$ such that $m_{k}=-\left\langle h_{i_{k}}, s_{\imath_{k-1}} \cdots s_{i_{1}} \lambda\right\rangle>0$ and $\mu=$ $s_{i l} \cdots s_{{ }_{11}} \lambda$ by Lemma 1.4. Then $e_{\imath l}^{m_{l}} \cdots e_{{ }_{11}}^{m_{1}}$ sends injectively $N_{\lambda}$ to $N_{\mu}$. Hence we have $\operatorname{dim} N_{\lambda} \leq \operatorname{dim} N_{\mu}$. Thus we obtain $\operatorname{dim} N_{\lambda}=\operatorname{dim} N_{\mu}$. Then the proposition follows from the following lemma.

Lemma 1.17. Let $M$ be a finite-dimensional integrable $U_{q}\left(\mathfrak{S l}_{2}\right)$-module and let $N$ be a vector subspace of $M$ stable by $e$ and $t$. If $\operatorname{dim} N_{\lambda}=\operatorname{dim} N_{s(\lambda)}$ for any $\lambda$ ( $s$ is the simple reflection), then $N$ is a $U_{q}\left(\mathfrak{s l}_{2}\right)$-submodule.

Proof. Any $u \in N_{\lambda}$ can be written

$$
u=\sum_{n} f^{(n)}{ }^{\prime}{ }_{n}
$$

with $e v_{n}=0$. Here $n$ ranges over $\{n \in \mathbb{Z} \geq 0 ; n+\langle h, \lambda\rangle \geq 0\}$.

Let us prove $U_{q}\left(\mathfrak{g l}_{2}\right) v_{n} \subset N$ by the descending induction on $c=\langle h, \lambda\rangle$. We have $e u=\sum_{n}[1+c+n] f^{(n-1)} v_{n}$. Hence the induction hypothesis implies $U_{q}\left(\mathfrak{g l}_{2}\right) v_{n}$ $\subset N$ for $n>0$. Hence we may assume that $e u=0$, and then $c \geq 0$. The surjectivity of $e^{c}: N_{s \lambda} \rightarrow N_{\lambda}$ implies the existence of $w \in N_{s \lambda}$ such that $u=e^{(c)} w$. Then $f w=0$ and $U_{q}\left(\mathfrak{B l}_{2}\right) u=U_{q}\left(\mathfrak{G l}_{2}\right) w$ is generated by $\left\{e^{n} w ; n \geq 0\right\} \subset N$.

Lemma 1.18. Let $M_{1}$ and $M_{2}$ be finite-dimensional $U_{q}^{\prime}(\mathfrak{g})$-modules and let 
$v_{1}$ and $v_{2}$ be non-zero weight vectors of $M_{1}$ and $M_{2}$. If $v_{1} \otimes v_{2}$ is extremal, then $v_{1}$ and $v_{2}$ are extremal and their weights are in the same Weyl chamber (in $\left.\mathrm{t}_{\mathrm{cl}}^{* 0}\right)$.

Proof. We may assume that wt $\left(v_{1} \otimes v_{2}\right)$ is dominant. Then for any $P \in$ $U_{q}^{\prime}(\mathfrak{b})$, we have

$$
P\left(v_{1} \otimes v_{2}\right)=v_{1} \otimes P v_{2}+\cdots .
$$

Hence the weights of $U_{q}^{\prime}(\mathfrak{b}) v_{2}$ is contained in wt $\left(v_{2}\right)+Q_{-}$. Since $U_{q}^{\prime}(\mathfrak{b}) v_{2}=U_{q}^{\prime}(\mathfrak{g})$ $v_{2}$ by Prop $1.16, v_{2}$ is an extremal vector with a dominant weight. Similary so is $v_{1}$.

\section{Conjecture}

We denote $\cup_{n>0} \mathbb{C}\left(\left(q^{1 / n}\right)\right)$ by $\bar{k}$ and $\cup_{n>0} \mathbb{C}\left[\left[q^{1 / n}\right]\right]$ by $\bar{A}$. Hence $\bar{k}$ is an algebraically closed field and $\bar{A}$ is a local ring. For $a, b \in \bar{k}^{x}=\bar{k} \backslash\{0\}$, we write $a$ $\leq b$ if $a / b \in \bar{A}$.

For $i \in I_{0}$, let $u_{\imath}$ denote the dominant extremal vector of $V\left(\pi_{\imath}\right)$.

Conjecture 1 . Let $i_{1}, \ldots, i_{l}$ be elements of $I_{0}$ and $a_{l}, \ldots, a_{1}$ non-zero elements of $\bar{k}$.

(1) If $a_{1} \leq \cdots \leq a_{l}$, then $V\left(\pi_{l_{1}}\right)_{a_{1}} \otimes \cdots \otimes V\left(\pi_{t_{l}}\right)_{a l}$ is generated by $u_{t_{1}} \otimes \cdots \otimes u_{\imath l}$ as a $U_{q}^{\prime}(\mathrm{g})_{\bar{k}}$-module.

(2) If $a_{1} \geq \cdots \geq a_{l}$, then any non-zero $U_{q}^{\prime}(\mathfrak{g})_{\bar{k}}$-submodule of $V\left(\pi_{11}\right)_{a_{1}} \otimes \cdots \otimes$ $v^{r}\left(\pi_{{ }_{11}}\right)_{a l}$ contains $u_{11} \otimes \cdots \otimes u_{\imath l}$.

Note that (1) and (2) are dual statements and therefore they are equivalent. One can compare (1) to the case of Verma modules and (2) to the case of the dual of Verma modules.

Let us discuss several consequences of this conjecture.

For $i, j \in I_{0}$, there is an intertwiner

$$
R_{i j}^{\text {nor }}(x, y): V\left(\pi_{l}\right)_{x} \otimes V\left(\pi_{j}\right)_{y} \rightarrow V\left(\pi_{j}\right)_{y} \otimes V\left(\pi_{i}\right)_{x} .
$$

We normalize this such that $R$ sends $u_{\imath} \otimes u_{j}$ to $u_{j} \otimes u_{\imath}$. Then we regard it as a rational function in $(x, y)$. Since it is homogeneous, its pole locus has the form $y / x=$ constant. We call it the normalized $R$-matrix. By Corollary 1.13 such an $R_{i j}^{\text {nor }}(x, y)$ is unique,

Corollary 2.1. If $a_{1} \leq a_{2}$, the normalized $R$-matrix $R_{i, j}^{\text {nor }}(x, y)$ does not have a pole at $(x, y)=\left(a_{1}, a_{2}\right)$.

Proof. Suppose that $R_{i, j}^{\text {nor }}(x, y)$ has a pole at $(x, y)=\left(a_{1}, a_{2}\right)$. Let $R^{\prime}$ be the non-zero $U_{q}^{\prime}(\mathrm{g})$-linear map $V\left(\pi_{i}\right)_{a_{1}} \otimes V\left(\pi_{j}\right)_{a_{2}} \rightarrow V\left(\pi_{j}\right)_{a_{2}} \otimes V\left(\pi_{i}\right)_{a_{1}}$ obtained after cancelling the poles of $R_{i, j}^{\text {nor }}(x, y)$. Then $R^{\prime}\left(u_{i} \otimes u_{j}\right)=0$, and hence $\operatorname{Im}\left(R^{\prime}\right)$ does 
not have weight $\pi_{i}+\pi_{j}$. On the other hand, Conjecture $1(2)$ implies that $\operatorname{Im}\left(R^{\prime}\right)$ contains $u_{0} \otimes u_{i}$, which is a contradiction. Hence $R_{\imath \jmath}^{\text {nor }}(x, y)$ has no pole at $\left(a_{1}\right.$, $\left.a_{2}\right)$.

Corollary 2.2. Let $K$ be a field extension of $k$, and $i_{1}, \ldots, i_{l} \in I_{0}, a_{1}, \ldots, a_{l} \in$ $K^{\times}=K \backslash\{0\}$.

(1) Assume that $R_{\nu, t u}^{\mathrm{nor}}(x, y)$ does not have a pole at $(x, y)=\left(a_{\nu}, a_{\mu}\right)$ for $1 \leq \nu<\mu$ $\leq l$. Then $V\left(\pi_{t_{1}}\right)_{a_{1}} \otimes \cdots \otimes V\left(\pi_{t l}\right)_{a l}$ is generated by $u_{{ }_{11}} \otimes \cdots \otimes u_{u_{1}}$ as a $U_{q}^{\prime}(\mathrm{g})_{K^{-}}$ module.

(2) Assume that $R_{i \nu, l_{\mu}}^{\text {nor }}(x, y)$ does not have a pole at $(x, y)=\left(a_{\nu}, a_{\mu}\right)$ for $1 \leq \mu<\nu$ $\leq l$. Then any non-zero $L_{q}^{\prime}(\mathrm{g})_{K}$-subnodule of $V\left(\pi_{l_{1}}\right)_{a_{1}} \otimes \cdots \otimes V\left(\pi_{l_{l}}\right)_{a_{l}}$ contains $u_{\imath_{1}}$ $\otimes \cdots \otimes u_{\imath \imath}$.

Proof. We may assume that $K$ is generated by $a_{1}, \cdots, a_{l}$ over $k$. Since $\bar{k}$ is an algebraically closed field with infinite transcendental dimension over $k$, there exists an embedding $K \rightarrow \bar{k}$. Hence we may assume $K=\bar{k}$.

Since the proof of (2) is similar, we shall only prove (1). We prove (1) by induction on the number of pairs $(\nu, \mu)$ with $\nu<\mu$ and $a_{\nu} \not \leq a_{\mu}$, which we denote by $n$. If $n=0$, the assertion follows immediately from Conjecture 1 . If $n>0$, take $\nu$ such that $a_{\nu} \not \leq a_{\nu+1}$. Hence $a_{\nu+1} \leq a_{\nu}$. Then Corollary 2.1 implies that $R_{l \nu+1, l \nu}^{\text {nor }}(x$, $y)$ does not have a pole at $(x, y)=\left(a_{\nu+1}, a_{\nu}\right)$. Since $R_{l \nu, l \nu+1}^{\text {nor }}(x, y)$ does not have a pole at $(x, y)=\left(a_{\nu}, a_{\nu+1}\right)$ by the assumption,

$$
R_{i \nu, l \nu+1}^{\text {nor }}\left(a_{\nu}, a_{\nu+1}\right): V\left(\pi_{i \nu}\right)_{a_{\nu}} \otimes V\left(\pi_{i_{\nu+1}}\right)_{a_{\nu+1}} \longrightarrow I^{I}\left(\pi_{i_{\nu+1}}\right)_{a_{\nu+1}} \otimes V\left(\pi_{i \nu}\right)_{a_{\nu}}
$$

and

$$
R_{i \nu+1, t \nu}^{\text {nor }}\left(a_{\nu+1}, a_{\nu}\right): V^{r}\left(\pi_{i \nu+1}\right)_{a_{\nu+1}} \otimes V\left(\pi_{i \nu}\right)_{a_{\nu}} \rightarrow V^{r}\left(\pi_{i \nu}\right)_{a_{\nu}} \otimes V\left(\pi_{i \nu+1}\right)_{a_{\nu+1}}
$$

are inverse of each other. Hence we can reduce the original case to the case where $\nu$ and $\nu+1$ are exchanged, in which $n$ is smaller than the original one by 1. Hence the induction proceeds.

Assume the condition (1) in the preceding Corollary 2.2. Let $R$ be the intertwiner

$$
R: V\left(\pi_{t_{1}}\right)_{a_{1}} \otimes \cdots \otimes V\left(\pi_{t l}\right)_{a l} \rightarrow V\left(\pi_{1 l}\right)_{a l} \otimes \cdots \otimes V\left(\pi_{t_{1}}\right)_{a_{1}}
$$

sending $u_{\imath_{1}} \otimes \cdots \otimes u_{\imath_{l}}$ to $u_{u_{1}} \otimes \cdots \otimes u_{u_{1}}$, obtained as the product of $R_{\imath_{\nu, l_{\mu}}}^{\text {nor }}\left(a_{\nu}, a_{\mu}\right)$ with $1 \leq \nu<\mu \leq l$.

Corollary 2.3. Lnder the condition (1) in Corollary 2.2. $\operatorname{Im}(R)$ is irreducible.

Note that the condition (1) is satisfied if $K=\bar{k}$ and $a_{1} \leq \cdots \leq a_{l}$, and hence we can apply the corollary in this case. 
Proof. By Corollary $2.2(1), \operatorname{Im}(R)$ is generated by the dominant extremal vector $u_{i_{l}} \otimes \cdots \otimes u_{i_{1}}$. Since any submodule of $\operatorname{Im}(R)$ contains the same vector by Corollary $2.2(2), \operatorname{Im}(R)$ is irreducible.

In fact, $\operatorname{Im}(R)$ is absolutely irreducible. Let us recall that, for a (not necessarily algebraically closed) field $K$ containing $k$, a $U_{q}^{\prime}(\mathfrak{g})_{K}$-module $M$ finite-dimensional over $K$ is called absolutely irreducible if the following equivalent conditions are satisfied.

(1) For some algebraically closed field $K^{\prime}$ containing $K, K^{\prime} \otimes_{K} M$ is an irreducible $U_{q}^{\prime}(\mathrm{g})_{K^{\prime}}$-module.

(2) For any algebraically closed field $K^{\prime}$ containing $K, K^{\prime} \otimes_{K} M$ is an irreducible $U_{q}^{\prime}(\mathfrak{g})_{K^{\prime}}$-module.

(3) $M$ is irreducible and End $_{U_{q}^{\prime}(g)_{K}}(M) \cong K$.

We denote by $m$ the maximal ideal $\cup_{n>0} q^{1 / n} \mathbb{C}\left[\left[q^{1 / n}\right]\right]$ of $\bar{A}$.

Corollary 2.4. For $a \in \bar{k}^{x}, y / x=a$ is a pole of $R_{z j}^{\text {nor }}(x, y)$ if and only if $a \in$ $\mathrm{m}$ and $V\left(\pi_{t}\right) \otimes V\left(\pi_{\jmath}\right)_{a}$ is reducible.

Proof. By Corollary 2.1, if $y / x=a$ is a pole of $R_{i j}^{\text {nor }}(x, y)$, then $a \in \mathfrak{m}$. By a similar argument to Corollary 2.1, the irreducibility of $V\left(\pi_{1}\right) \otimes V\left(\pi_{j}\right)_{a}$ implies that $y / x=a$ is not a pole of $R_{\imath j}^{\text {nor }}(x, y)$. Now assume that $y / x=a \in m$ is not a pole of $R_{i j}^{\text {nor }}(x, y)$. Since $R_{j_{l}}^{\text {nor }}(a, 1)$ is well defined, $R_{i j}^{\text {nor }}(1, a)$ is invertible. Hence $V\left(\pi_{2}\right) \otimes V\left(\pi_{j}\right)_{a}$ is irreducible by Corollary 2.3.

Corollary 2.5. Let $K$ be an algebraically closed field containing $k$. If $M$ and $M^{\prime}$ are irreducible finite-dimensional integrable $U_{q}^{\prime}(\mathfrak{g})_{K}$-modules, then $M \otimes M_{z}^{\prime}$ is an irreducible $L_{q}^{\prime}(\mathrm{g})_{K^{-}}$module except finitely many $z \in K$.

Proof. Let $M\left(\operatorname{resp} . M^{\prime}\right)$ be the irreducible subquotient of $V\left(\pi_{i_{1}}\right)_{a_{1}} \otimes \cdots \otimes V$ $\left(\pi_{i m}\right)_{a m}\left(\right.$ resp. $\left.V\left(\pi_{i 1}\right)_{a 1} \otimes \cdots \otimes V\left(\pi_{i^{\prime} m^{\prime}}\right)_{a_{m^{\prime}}}\right)$ such that $R_{i \nu, i \mu}^{\text {nor }}(x, y)\left(\right.$ resp. $\left.R_{i \nu, i_{i \mu}}^{\text {nor }}(x, y)\right)$ does not have a pole at $(x, y)=\left(a_{\nu}, a_{\mu}\right)\left(\right.$ resp. $\left.(x, y)=\left(a_{\nu}^{\prime}, a_{\mu}^{\prime}\right)\right)$ for $1 \leq \nu<\mu \leq m$ (resp. $1 \leq \nu<\mu \leq m^{\prime}$ ). Then Corollary 2.3 implies that $M$ is isomorphic to the image of the $R$-matrix

$$
R: V \longrightarrow W,
$$

where $V=V\left(\pi_{i_{1}}\right)_{a_{1}} \otimes \cdots \otimes V\left(\pi_{i m}\right)_{a_{m}}$ and $W=V\left(\pi_{t_{m}}\right)_{a_{m}} \otimes \cdots \otimes V\left(\pi_{t_{1}}\right)_{a_{1}}$. Similarly $M^{\prime}$ is isomorphic to the image of

$$
R^{\prime}: V^{\prime} \longrightarrow W^{\prime}
$$

where $V^{\prime}=V\left(\pi_{t 1}\right)_{a 1} \otimes \cdots \otimes V\left(\pi_{i m^{\prime}}\right)_{a^{\prime} m^{\prime}}$ and $W^{\prime}=V\left(\pi_{i^{\prime} m^{\prime}}\right)_{a^{\prime} m^{\prime}} \otimes \cdots \otimes V\left(\pi_{i_{1}^{\prime}}\right)_{a_{1}^{\prime}}$. If $z$ is generic, $R_{i \nu, l^{\prime} \nu^{\prime}}^{\text {nor }}(x, y)$ does not have a pole at $(x, y)=\left(a_{\nu}, z a_{\nu^{\prime}}^{\prime}\right)$ and $R_{\nu^{\prime}, i \nu}^{\text {nor }}(x, y)$ does not have a pole at $(x, y)=\left(z a_{\nu^{\prime}}^{\prime}, a_{\nu}\right)$. Hence the $R$-matrix $W \otimes W_{z}^{\prime} \rightarrow W_{z}^{\prime} \otimes W$ is an isomorphism. Hence the image of the composition 


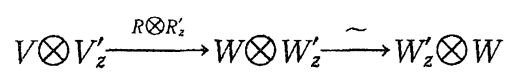

is isomorphic to $M \otimes M_{z}^{\prime}$ and it is irreducible by Corollary 2.3 .

Hence the intertwiner $M \otimes M_{z}^{\prime} \rightarrow M_{z}^{\prime} \otimes M$ is unique up to constant.

We give a conjecture on the poles of the $R$-matrices.

Conjecture 2. For $i, j \in I_{0}$, the pole of the normalized $R$-matrix $R_{i j}^{\text {nor }}(x, y)$ has the form $y / x= \pm q^{n}$ for $n \in \gamma^{-1} Z$ with $0<n \leq(\delta, \rho)$ except $D_{4}^{(3)}$ (where $\gamma$ is defined in $(1.2))$. In the $D_{4}^{(3)}$ case the third root of unity appears in the coefficients.

As seen in the appendix, this is true for $A_{n}^{(1)}$ and $C_{n}^{(1)}$.

We can also ask if the following statements are true.

(2.2) $R_{l j}^{\text {nor }}(x, y)$ has only a simple pole.

(2.3) If $(x, y)=(a, b)$ is a pole of $R_{i j}^{\text {nor }}(x, y)$, then the kernel of $R_{j t}^{\text {nor }}(b, a)$ : $V\left(\pi_{j}\right)_{b} \otimes V\left(\pi_{l}\right)_{a} \rightarrow V^{r}\left(\pi_{\imath}\right)_{a} \otimes V^{r}\left(\pi_{J}\right)_{b}$ is irreducible.

\section{Reduction of the Conjecture}

In this section we shall prove that Conjecture 1 follows from Conjecture 3 below. Let $\mathrm{m}=\cup_{n>0} q^{1 / n} \mathrm{C}\left[\left[q^{1 / n}\right]\right]$ be the maximal ideal of $\bar{A}$.

Conjecture 3. For every $i \in I_{0}$, there exist $N \in \mathbb{N}, b_{1}, \ldots, b_{N}, c_{1}, \ldots, c_{N} \in \mathfrak{m} \backslash\{0\}$. $s_{1}, \ldots, s_{N}, t_{1}, \ldots, t_{N} \in I_{0}$, an irreducible finite-dimensional $U_{q}^{\prime}(\mathrm{g})_{\bar{k}}$-module $W_{\mu}$ and a $U_{q}^{\prime}(\mathrm{g})_{\bar{k}}$-linear map $\varphi_{\mu}: V\left(\pi_{\imath}\right) \otimes V\left(\pi_{s \mu}\right)_{b \mu} \rightarrow V\left(\pi_{t \mu}\right)_{c_{\mu}} \otimes W_{\mu}$ for $\mu$ with $1 \leq \mu \leq N$,

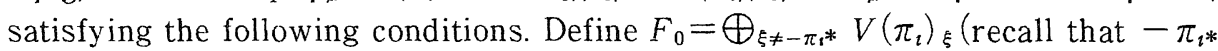
is the lowest weight vector of $\left.V\left(\pi_{1}\right)\right)$ and $F_{\mu}=\left\{v \in F_{\mu-1} \mid \varphi_{\mu}\left(v \otimes u_{s_{\mu}}\right)=0\right\}$ for 0 $<\mu \leq N$.

(1) $F_{N}=\bar{k} u_{1}$.

(2) $\varphi_{\mu}\left(F_{\mu-1} \otimes u_{s_{\mu}}\right) \subset V\left(\pi_{t \mu}\right)_{c \mu} \otimes w_{\mu}$.

(3) $V\left(\pi_{s u}\right)_{b \mu}$ is not isomorphic to $V\left(\pi_{t \mu}\right)_{c_{\mu}}$.

(4) $V\left(\pi_{s \mu}\right)_{b \mu}$ is not a component of $W_{\mu}$ (see Definition 1.3).

Here $u_{s \mu}$ and $w_{\mu}$ are dominant extremal vectors of $V\left(\pi_{s \mu}\right)_{b \mu}$ and $W_{\mu}$, respectively.

Let us show that Conjecture 3 implies Conjecture $1(2)$.

For $a_{1}, \ldots, a_{p} \in \bar{k}^{\times}$, let $P\left(a_{1}, \ldots, a_{p}\right)$ denote the following statement.

$P\left(a_{1}, \ldots, a_{p}\right):$ For indeterminates $x_{1}, \cdots, x_{l}$, any dominant extremal vector of the

$U_{q}^{\prime}(\mathfrak{g})_{\bar{k}^{\prime}\left(x_{1}, x_{l}\right)}$-module $V\left(\pi_{j_{1}}\right)_{x_{1}} \otimes \cdots \otimes V\left(\pi_{\mu_{l}}\right)_{x_{l}} \otimes V\left(\pi_{i_{1}}\right)_{a_{1}} \otimes \cdots \otimes V\left(\pi_{i_{p}}\right)_{a_{p}}$

is a constant multiple of $u_{j_{1}} \otimes \cdots \otimes u_{j_{l}} \otimes u_{u_{1}} \otimes \cdots \otimes u_{u_{p}}$.

Assuming Conjecture 3, we shall prove the following lemma. 
Lemma 3.1. If $a_{1, \ldots,} a_{p} \in \bar{k}^{\times}$satisfy $a_{1} \geq \cdots \geq a_{p}$, then $P\left(a_{1}, \ldots, a_{p}\right)$ holds.

Since any non-zero finite-dimensional module contains a dominant extremal vector, this lemma implies Conjecture $1(2)$. We shall prove this lemma by induction on $p$. First assume $p \geq 1$. Then $P\left(a_{1}, \ldots, a_{p-1}\right)$ holds by the hypothesis of induction. Set $K=\bar{k}\left(x_{1}, \ldots, x_{l}\right)$. Let $x$ be another indeterminate. By the existence of $R$-matrix, $V\left(\pi_{j_{1}}\right)_{x_{1}} \otimes \cdots \otimes V\left(\pi_{\imath_{l}}\right)_{x_{1}} \otimes V\left(\pi_{l_{1}}\right)_{a_{1}} \otimes \cdots \otimes V\left(\pi_{i_{p-1}}\right)_{a_{p-1}} \otimes V\left(\pi_{i_{p}}\right)_{x}$ is isomorphic to $V\left(\pi_{j_{1}}\right)_{x_{1}} \otimes \cdots \otimes V\left(\pi_{l_{l}}\right)_{x_{l}} \otimes V\left(\pi_{i p}\right)_{x} \otimes V\left(\pi_{i_{1}}\right)_{a_{1}} \otimes \cdots \otimes V\left(\pi_{i p-1}\right)_{a_{p-1}}$. Hence $P\left(a_{1}, \cdots, a_{p-1}\right)$ implies that a dominant extremal vector of the $U_{q}^{\prime}(g)_{K(x)^{-}}$ module $V\left(\pi_{j_{1}}\right)_{x_{1}} \otimes \cdots \otimes V\left(\pi_{\jmath_{l}}\right)_{x_{l}} \otimes V\left(\pi_{t_{1}}\right)_{a_{1}} \otimes \cdots \otimes V\left(\pi_{i_{p-1}}\right)_{a_{p-1}} \otimes V\left(\pi_{i_{p}}\right)_{x}$ is a constant multiple of $u_{j_{1}} \otimes \cdots \otimes u_{j_{l}} \otimes t_{t_{1}} \otimes \cdots \otimes u_{i_{p}}$. Then it follows that a dominant extremal vector of $U_{q}^{\prime}(\mathrm{g})_{K}$-module $V\left(\pi_{j_{1}}\right)_{x_{1}} \otimes \cdots \otimes V\left(\pi_{j_{l}}\right)_{x_{l}} \otimes V\left(\pi_{i_{1}}\right)_{a_{1}} \otimes \cdots \otimes$ $V\left(\pi_{t_{p-1}}\right)_{a_{p-1}} \otimes V\left(\pi_{i_{p}}\right)_{z}$ is a constant multiple of $u_{j_{1}} \otimes \cdots \otimes u_{j_{l}} \otimes u_{u_{1}} \otimes \cdots \otimes_{u_{i_{p}} \text { except }}$ for finitely many $z \in k$. This means that $P\left(a_{1}, \ldots, a_{p-1}, z\right)$ holds except finitely many $z \in \bar{k}$. Arguing by induction on the order of the zero of $a_{p}$, we may assume from the beginning

$$
P\left(a_{1}, \ldots, a_{p-1}, z\right) \text { holds for any } z \in m a_{p} \backslash\{0\} .
$$

Let $v$ be a dominant extremal vector of $U_{q}^{\prime}(\mathfrak{g})_{K}$-module $V\left(\pi_{j_{1}}\right)_{x_{1}} \otimes \cdots \otimes V\left(\pi_{l_{l}}\right)_{x_{l}} \otimes$ $V\left(\pi_{i_{1}}\right)_{a_{1}} \otimes \cdots \otimes V\left(\pi_{i_{p}}\right)_{a_{p}}$. We shall prove that $v$ is a constant multiple of $u_{j_{1}} \otimes \cdots$ $\otimes u_{u_{l}} \otimes u_{u_{1}} \otimes \cdots \otimes_{u_{i p}}$

We have $\varphi_{0}: V\left(\pi_{i p}\right) \otimes V\left(\pi_{i p}\right)_{y} \rightarrow \bar{k}$ with $y=(-1)^{\left.\prime \delta, \rho^{\nu}\right)} q^{(\delta, \rho)}$ by (1.6). Set $V^{\prime}$ $=V\left(\pi_{j_{1}}\right)_{x_{1}} \otimes \cdots \otimes V\left(\pi_{\jmath_{l}}\right)_{x_{l}} \otimes V\left(\pi_{i_{1}}\right)_{a_{1}} \otimes \cdots \otimes V\left(\pi_{l_{p-1}}\right)_{a_{p-1}}$. Then we have a morphism

$$
\operatorname{id}_{V^{\prime}} \otimes\left(\varphi_{0}\right)_{a_{p}}: V^{\prime} \otimes V\left(\pi_{i p}\right)_{a_{p}} \otimes V\left(\pi_{i p} *\right)_{a p y} \rightarrow V^{\prime}
$$

Lemma 3.2. We have $\left(\mathrm{id}_{V^{\prime}} \otimes\left(\varphi_{0}\right)_{a_{p}}\right)\left({ }_{v}, \otimes u_{i_{p} *}\right)=0$.

Proof. Assume that $w=\left(\operatorname{id}_{V^{\prime}} \otimes\left(\varphi_{0}\right)_{a_{p}}\right)\left(v_{r} \otimes u_{i_{p} *}\right) \neq 0$. Then $w$ is a dominant extremal vector of $V^{\prime}$. Hence $w$ is equal to $u_{j_{1}} \otimes \cdots \otimes u_{u_{l}} \otimes_{u_{u_{1}}} \otimes \cdots \otimes u_{t_{p-1}}$ up to a constant multiple by $P\left(a_{1} \ldots, a_{p-1}\right)$. Therefore Theorem 1.2 implies that

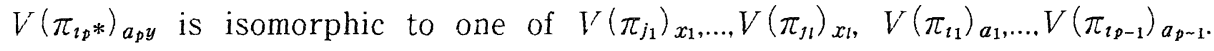
This is a contradiction since $y \in q A$.

Since $F_{0}=\left\{w \in V\left(\pi_{\imath_{p}}\right) ; \varphi_{0}\left(w \otimes u_{\imath p^{*}}\right)=0\right\}$, we have $v \in V^{\prime} \otimes\left(F_{0}\right)_{a_{p}}$. Now we shall show $\imath^{\prime} \in V^{\prime} \otimes\left(F_{\mu}\right)_{a_{p}}$ by induction on $\mu$. Applying Conjecture 3 with $i=i_{p}$, we have $U_{q}^{\prime}(\mathrm{g})$-linear maps $\varphi_{\mu}: V\left(\pi_{t_{p}}\right) \otimes V\left(\pi_{s_{\mu}}\right)_{b_{\mu}} \rightarrow V\left(\pi_{t_{\mu}}\right)_{c_{\mu}} \otimes W_{\mu}$ for $1 \leq \mu \leq N$ satisfying the conditions (1) - (4) in Conjecture 3 . Then this induces a homomorphism

$$
\operatorname{id}_{V^{\prime}} \otimes\left(\varphi_{\mu}\right)_{a_{p}}: V^{\prime} \otimes V\left(\pi_{i_{p}}\right)_{a_{p}} \otimes V\left(\pi_{s_{\mu}}\right)_{a_{p} b_{\mu}} \rightarrow V^{\prime} \otimes V\left(\pi_{t_{\mu}}\right)_{a_{p} c_{\mu}} \otimes\left(W_{\mu}\right)_{a_{p}}
$$

Suppose that $\imath^{\prime} \in V^{\prime} \otimes\left(F_{\mu-1}\right)_{a_{p}}$, which is the case when $\mu=1$.

Lemma 3.3. We have $\left(\operatorname{id}_{V^{\prime}} \otimes\left(\varphi_{\mu}\right)_{a_{p}}\right)\left(v \otimes u_{s_{\mu}}\right)=0$. 
Proof. The proof is similar to the one of the preceding lemma. Suppose that $w=\left(\operatorname{id}_{V^{\prime}} \otimes\left(\varphi_{\mu}\right)_{a_{p}}\right)\left(v \otimes u_{s_{\mu}}\right)$ is not zero. Write $w$ as $v^{\prime \prime} \otimes w_{\mu}$ in virtue of the condition (2) in Conjecture 3, where $v^{\prime \prime}$ is a non-zero vector of $V^{\prime} \otimes V\left(\pi_{t_{\mu}}\right)_{a_{p} c_{\mu}}$. Since $v \otimes u_{s \mu}$ is extremal, so is $v^{\prime \prime} \otimes w_{\mu}$. Hence $v^{\prime \prime}$ is a dominant extremal vector by Lemma 1.18. Since $a_{p} c_{\mu} \in \mathfrak{m} a_{p}$, the property $P\left(a_{1}, \ldots, a_{p-1}, a_{p} c_{\mu}\right)$ holds by (3.1), and hence $v^{\prime \prime}$ is a nonzero scalar multiple of $u_{j_{1}} \otimes \cdots \otimes u_{\jmath_{l}} \otimes u_{i_{1}} \otimes \cdots \otimes u_{i_{p}} \otimes u_{t_{\mu}}$. Then Theorem 1.2 implies that $V\left(\pi_{s_{\mu}}\right)_{a_{p} b_{\mu}}$ is isomorphic to one of $V\left(\pi_{p_{1}}\right)_{x_{1}}$,

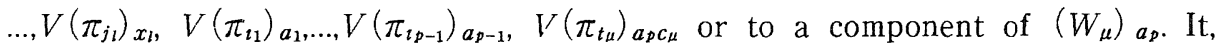
however, is not the case because of the conditions (3). (4) in Conjecture 3 and $a_{p} b_{\mu} \in \mathfrak{m} a_{p}$.

By this we have $v \in V^{r \prime} \otimes\left(F_{\mu}\right)_{a_{p}}$. Applying this process successively, we obtain $v \in V^{\prime} \otimes\left(F_{N}\right)_{a_{p}}$. Hence we have $v \in V^{\prime} \otimes u_{\imath_{p}}$ by the condition (1) in Conjecture 3. Write $v$ as $v^{\prime} \otimes u_{\imath_{p}}$, where $v^{\prime}$ is a nonzero vector of $V^{\prime}$. Lemma 1.18 implies that $v^{\prime}$ is dominant and extremal. Therefore $\imath^{\prime}$ is a nonzero scalar multiple of $u_{1_{1}} \otimes \cdots \otimes u_{l_{l}} \otimes u_{t_{1}} \otimes \cdots \otimes u_{i_{p-1}}$ by the induction hypothesis on $p$. We have deduced the $p$ case from the $p-1$ case.

It remains to prove $p=0$ case, which follows from the following lemma.

Lemma 3.4. Any dominant extremal vector of the $U_{q}^{\prime}(\mathfrak{g})_{\bar{k}\left(x_{1}, x_{t}\right)}$-module $V\left(\pi_{l_{1}}\right)_{x_{1}} \otimes \cdots \otimes V\left(\pi_{1 l}\right)_{x_{l}}$ is a constant multiple of $u_{u_{1}} \otimes \cdots \otimes u_{1 l}$. Here $x_{1}, \ldots, x_{l}$ are indeterminates.

Proof. It is enough to prove the assertion with $x_{1}=\cdots=x_{l}=1$. Let $l^{\prime}$ denote $V\left(\pi_{t_{1}}\right) \otimes \cdots \otimes V\left(\pi_{\imath l}\right)$. By Corollary 1.13, $V$ is irreducible. Suppose now that $V$ has a dominant extremal vector $v^{\prime}$ that is not a constant multiple of $u_{u_{1}} \otimes$

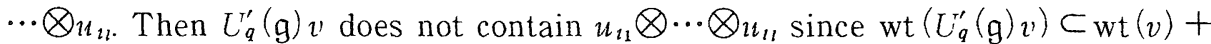
$\sum_{t \in I_{0}} \mathbb{Z}_{\leq 0} \operatorname{cl}\left(\alpha_{t}\right)$, which is a contradiction.

Thus we have proved

Proposition 3.5. Conjecture 3 implies Conjecture 1.

\section{Proof of Conjecture 3 for $\mathbb{A}_{n}^{(1)}$ and $\mathbb{C}_{n}^{(1)}$}

In this section, we shall prove the following theorem.

Theorem 4.1. Conjecture 3 holds if $\mathrm{g}$ is $A_{n}^{(1)}$ or $C_{n}^{(1)}$.

4.1. $A_{n-1}^{(1)}$ Case. For the fundamental representations of $U_{q}^{\prime}\left(\widehat{\mathfrak{B l}}_{n}\right)$, see Appendix B.1. We identify crystal bases of the fundamental representations with the corresponding global bases.

Let us prove Conjecture 3 .

Since the $i=n-1$ case can be reduced to the case $i=1$ by the Dynkin diagram automorphism, we assume $1 \leq i<n-1$. Set $N=i$. For $1 \leq \mu \leq N=i$, take 
$s_{\mu}=\mu, t_{\mu}=i+1, b_{\mu}=(-q)^{i-\mu+2}, c_{\mu}=-q, W_{\mu}=V\left(\pi_{\mu-1}\right)_{(-q))^{t-\mu+1}}$ and define $\varphi_{i, \mu}$ : $V\left(\pi_{\imath}\right) \otimes V\left(\pi_{\mu}\right)_{(-q)^{\imath-\mu+2}} \rightarrow V\left(\pi_{i+1}\right)_{-q} \otimes W_{\mu}$ as the composition (see Lemma B. 1):

$$
\begin{gathered}
V\left(\pi_{i}\right) \otimes V\left(\pi_{\mu}\right)_{(-q) x-\mu+2} \stackrel{V\left(\pi_{i}\right) \otimes\left(i_{1, \mu-1}\right)_{(-q)^{r-\mu+2}}}{\longrightarrow} V\left(\pi_{i}\right) \otimes V\left(\pi_{1}\right)_{(-q)^{t+1}} \otimes W_{\mu} \\
\downarrow\left(p_{h}, 1\right)_{-q} \otimes W_{\mu} \\
V\left(\pi_{i+1}\right)_{-q} \otimes W_{\mu} .
\end{gathered}
$$

Then it is easy to check that Conjecture 3 holds with

$$
F_{\mu}=\bigoplus_{\mu<a_{\mu+1}<\cdots<a_{1} \leq n} k\left(1, \ldots, \mu, a_{\mu+1}, \ldots, a_{2}\right) .
$$

4.2. $C_{n}^{(1)}$ Case. For the fundamental representations of $U_{q}^{\prime}\left(C_{n}^{(1)}\right)$. see Appendix C.1.

For $1 \leq i<n$, let $p_{i}: V\left(\pi_{i}\right) \otimes V\left(\pi_{1}\right)_{\left(-q_{s}\right)^{i+1}} \longrightarrow V\left(\pi_{i+1}\right)_{-q_{s}}$ be $\left(p_{i, 1}\right)_{-q_{s}}$. Let $p_{n}$ : $V\left(\pi_{n}\right) \otimes V\left(\pi_{1}\right)_{\left(-q_{s}\right)^{n+3}} \longrightarrow V\left(\pi_{n-1}\right)_{-q_{s}}$ be the composition

$$
\begin{aligned}
& \left.V\left(\pi_{n}\right) \otimes V\left(\pi_{1}\right)_{\left(-q_{s}\right)}\right)^{n+3} \\
& \imath_{n-11} \otimes V\left(\pi_{1}\right)_{\left(-q_{3}\right)^{n+3}} \downarrow \\
& V\left(\pi_{n-1}\right)_{-q_{s}} \otimes V\left(\pi_{1}\right)_{\left(-q_{s}\right)^{1-n}} \otimes V\left(\pi_{1}\right)_{(-q s)} \stackrel{V\left(\pi_{n-1}\right)_{-q s} 3 \mathrm{tr}}{\longrightarrow} V\left(\pi_{n-1}\right)_{-q_{s} .}
\end{aligned}
$$

Here $\operatorname{tr}$ is given in (C.1).

For $1 \leq_{i} \leq_{n}-1$, set $N=i$. For $1 \leq \mu \leq N=i$, we set $s_{\mu}=\mu, t_{\mu}=i+1, b_{\mu}=$ $\left(-q_{s}\right)^{2-\mu+2}, c_{\mu}=-q_{s}$ and $W_{\mu}=V\left(\pi_{\mu-1}\right)_{\left(-q_{s}\right)^{t-\mu+1}}$. We define $\varphi_{i, \mu}: V\left(\pi_{\imath}\right) \otimes$ $V\left(\pi_{\mu}\right)_{\left(-q_{s}\right)^{t-\mu+2}} \longrightarrow V\left(\pi_{i+1}\right)_{-q_{s}} \otimes W_{\mu}$ as the composition:

$$
\begin{gathered}
V\left(\pi_{l}\right) \otimes V\left(\pi_{\mu}\right)_{\left(-q_{s}\right)^{1-\mu+2}} \stackrel{V^{\prime}\left(\pi_{t}\right) \otimes\left(i_{1, \mu-1}\right)_{\left(-q_{s}\right)^{\prime-4+2}}}{\longrightarrow} V\left(\pi_{l}\right) \otimes V\left(\pi_{\mathbf{1}}\right)_{\left(-q_{s}\right)^{i+1}} \otimes W_{\mu} \\
\downarrow p_{i} \otimes W_{\mu} \\
V\left(\pi_{\imath+1}\right)_{-q_{s}} \otimes W_{\mu} .
\end{gathered}
$$

Note that $b_{\mu}, c_{\mu} \in q_{\mathrm{s}} A$.

For $i=n$, set $N=n$. For $1 \leq \mu \leq n$, we set $s_{\mu}=\mu, t_{\mu}=n-1, b_{\mu}=\left(-q_{s}\right)^{n-\mu+4}, c_{\mu}$

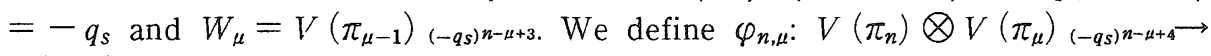
$V\left(\pi_{i+1}\right)_{-q s} \otimes W_{\mu}$ as the composition;

$$
\begin{gathered}
V\left(\pi_{n}\right) \otimes V\left(\pi_{\mu}\right)_{\left(-q_{s}\right) n-\mu+4} \stackrel{V\left(\pi_{n}\right) \otimes\left(i_{1, \mu-1}\right)_{\left(-q_{s}\right)^{n \mu+4}}}{\longrightarrow} V\left(\pi_{n}\right) \otimes V\left(\pi_{1}\right)_{\left(-q_{s}\right) n+3} \otimes W_{\mu} \\
\downarrow p_{n} \otimes W_{\mu} \\
V\left(\pi_{n-1}\right)_{-q_{s}} \otimes W_{\mu} .
\end{gathered}
$$

Note that $b_{\mu}, c_{\mu} \in q_{s} A$.

Then we have 


$$
F_{\mu}=\left\{v \in V\left(\pi_{i}\right) ; p_{i}(v \otimes G(j))=0 \quad \text { for } 1 \leq j \leq \mu\right\} .
$$

Then Conjecture 3 easily follows from the following lemma.

Lemma 4.2. Fix $1 \leq i \leq n$. Then

$$
\left\{v \in V\left(\pi_{i}\right) ; p_{i}(v \otimes G(j))=0 \text { for all } 1 \leq j \leq i\right\}=k G(1, \cdots, i) .
$$

Proof. Let $E$ be the left-hand-side of (4.4). Then $E$ is invariant by $e_{k}$ for any $k \in I_{0}$. Let us prove $E_{\lambda}=0$ by induction on the weight $\lambda \neq \pi_{i}$. We can easily check the assertion when $\lambda=\pi_{i}-\alpha_{i}$, since $V\left(\pi_{i}\right)_{\lambda}=k f_{i} u_{i}$. If a weight $\lambda$ of $V\left(\pi_{i}\right)$ is not $\pi_{i}-\alpha_{\imath}$, then $\lambda+\alpha_{k} \neq \pi_{\imath}$ for any $k \in I_{0}$. Therefore any $v \in E_{\lambda}$ satisfies $e_{k} v=$ 0 for all $k \in I_{0}$ by the induction hypothesis. This implies $v=0$.

\section{Appendix A. Universal $\mathbf{R}$-Matrix}

In this appendix we shall calculate the normalized and universal $R$-matrices of $U_{q}^{\prime}(\mathrm{g})$ for the fundamental representations following a variant of the recipe of Frenkel-Reshetikhin [8] in the $A_{n-1}^{(1)}$ and $C_{n}^{(1)}$ cases.

Let us choose the following universal $R$-matrix. Let us take a base $P_{\nu}$ of $U_{q}^{+}(\mathrm{g})$ and $Q_{\nu}$ of $U_{q}^{-}(\mathrm{g})$ dual to each other with respect a suitable coupling between $U_{q}^{+}(\mathfrak{g})$ and $U_{q}^{-}(\mathfrak{g})$. Then for $U_{q}^{\prime}(\mathfrak{g})$-modules $M$ and $N$ define

$$
R_{M N}^{\mathrm{unv}}\left(u \otimes_{\nu}\right)=q^{(\mathrm{wt}(u), \mathrm{wt}(v))} \sum_{\nu} P_{\nu} v \otimes Q_{\nu} u
$$

so that $R_{M N}^{\text {univ }}$ gives a $U_{q}^{\prime}(\mathrm{g})$-linear homomorphism from $M \otimes N$ to $N \otimes M$ provided the infinite sum has a meaning. If $M$ and $N$ are finite-dimensional integrable

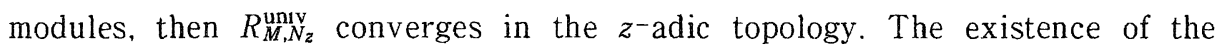
universal $R$-matrix for $(M, N)$ is proved by [6] (see also [18]). For a scalar $a$, the composition

$$
\left(R_{M, N}^{\text {univ }}\right)_{a}: M_{a} \otimes N_{a} \cong(M \otimes N)_{a} \rightarrow(N \otimes M)_{a} \cong N_{a} \otimes M_{a}
$$

is equal to $R_{M a, N_{a}}^{\text {univ }}$, and we sometimes confuse them.

For irreducible $U_{q}^{\prime}(\mathrm{g})$-modules $M$ and $N$, let us denote by $R_{M N}^{\text {nor }}(z)$ the $R$ matrix $M \otimes N_{z} \rightarrow N_{z} \otimes M$ normalized by $R_{M N}^{\text {nor }}(z)(u \otimes v)=v \otimes u$ for dominant extremal vectors $u$ (resp. $v$ ) of $M$ (resp. $N$ ). Let $d_{M N}(z)$ be the denominator of $R_{M N}^{\text {nor }}(z)$. Namely $c(z) \in k\left[z, z^{-1}\right]$ is divisible by $d_{M N}(z)$ if and only if $c(z) R_{M N}^{\text {nor }}(z)$ has no poles. Then $d_{M N}(z)$ is uniquely determined modulo $k\left[z, z^{-1}\right]^{\times}$. Here $k\left[z, z^{-1}\right]^{\times}$is the set of invertible elements of $k\left[z, z^{-1}\right]$. Hence

$$
k\left[z, z^{-1}\right]^{\times}=\left\{c z^{n} ; n \in \mathbb{Z}, c \in k \backslash\{0\}\right\} .
$$

Since the intertwiner from $M \otimes N_{z}$ to $N_{z} \otimes M$ is unique up to a constant multiple 
by Corollary 2.5, we can write
(A.3)
$R_{M N}^{\text {univ }}(z)=a_{M N}(z) R_{M N}^{\text {nor }}(z)$.

If $\lambda$ and $\mu$ are the dominant extremal weight of $M$ and $N$ respectively, we have

$$
a_{M N}(z) \in q^{(\lambda, \mu)}(1+z k[[z]]) .
$$

For $i, j \in I_{0}$, we denote $R_{i j}^{\text {univ }}(z)=R_{V\left(\pi_{i}\right) V(\pi,)}^{\text {univ }}(z), R_{i j}^{\text {nor }}(z)=R_{V\left(\pi_{t}\right) V(\pi,)}^{\text {nor }}(z), a_{i j}(z)$ $=a_{V\left(\pi_{i}\right) V(\pi,)}(z)$ and $d_{i j}(z)=d_{V(\pi i) V(\pi,)}(z)$.

For a finite-dimensional $U_{q}^{\prime}(\mathrm{g})$-module $M$, let $M^{*}$ be the left dual of $M$ and ${ }^{*} M$ the right dual of $M$. Hence we have

$$
\begin{array}{ll}
M^{*} \otimes M \stackrel{\operatorname{tr}}{\longrightarrow} k & k \stackrel{\iota}{\longrightarrow} M \otimes M^{*} \\
M \otimes * M \stackrel{\operatorname{tr}}{\longrightarrow} k & k \stackrel{\iota}{\longrightarrow} M \otimes M .
\end{array}
$$

We have

$$
M^{* *} \cong M_{q^{-2(\delta \rho)}} \text { and } \quad * * M \cong M_{q^{2(\delta \rho)}}
$$

We have

$$
V\left(\pi_{\imath}\right)^{*} \cong V\left(\pi_{1 *}\right)_{p *-1} \text { and } \quad * V\left(\pi_{\imath}\right) \cong V\left(\pi_{\imath *}\right)_{p *}
$$

where $p^{*}=(-1)^{\left(c, \rho^{v}\right)} q^{(\delta, \rho)}$.

Let $a \mapsto \bar{a}$ be the ring automorphism of $U_{q}^{\prime}(\mathfrak{g})$ given by $\bar{q}=q^{-1},\left(e_{2}\right)^{-}=e_{2},\left(f_{t}\right)^{-}$ $=f_{2}, q(h)^{-}=q(-h)$. For a $U_{q}^{\prime}(g)$-module $M$, let $M^{-}$be the $U_{q}^{\prime}(g)$-module whose underlying vector space is $M$ with the new action $U_{q}^{\prime}(\mathfrak{g}) \stackrel{-}{\rightarrow} U_{g}^{\prime}(\mathfrak{g}) \rightarrow$ End $(M)$. Then $(M \otimes N)^{-} \cong N^{-} \otimes M^{-}$and $V^{-}\left(\pi_{l}\right)^{-} \cong V\left(\pi_{\imath}\right)$. Hence we have

$$
d_{l \imath}(z) \equiv d_{\imath j}\left(z^{-1}\right)^{-} \bmod k\left[z, z^{-1}\right]^{\times} .
$$

The conjecture 2 implies

$$
d_{\jmath l}(z) \equiv d_{\imath \jmath}(z) \bmod k\left[z, z^{-1}\right]^{\times} .
$$

Proposition A.1. For irreducible finite-dimensional integrable $U_{q}^{\prime}(\mathfrak{g})$ modules $V$ and $W$, we have

$$
a_{V, W}(z) a_{V, W}(z) \equiv \frac{d_{V W}(z)}{d_{W, * V}\left(z^{-1}\right)} \bmod k\left[z, z^{-1}\right]^{\times} .
$$

Proof. For a $U_{q}^{\prime}(\mathrm{g})$-linear homomorphism $\phi: V \otimes W_{z} \rightarrow W_{z} \otimes V$, we shall define $\mathscr{T}_{r}(\phi): W_{z} \otimes * V \rightarrow * V \otimes W_{z}$ as the composition

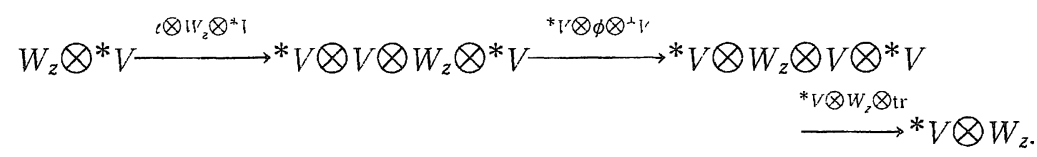


The correspondence $\phi \mapsto \mathscr{T}_{r}(\phi)$ gives an isomorphism

$$
\operatorname{Hom}\left(V \otimes W_{z}, W_{z} \otimes V\right) \stackrel{\sim}{\longrightarrow} \operatorname{Hom}\left(W_{z} \otimes^{*} V,{ }^{*} V \otimes W_{z}\right) .
$$

If we consider them as modules over $k\left[z, z^{-1}\right]$, then Hom $\left(V \otimes W_{z}, W_{z} \otimes V\right)$ is generated by $d_{V W}(z) R_{V W}^{\text {nor }}(z)$, and Hom $\left(W_{z} \otimes * V,{ }^{*} V \otimes W_{z}\right)$ is generated by $d_{W, * V}$ $\left(z^{-1}\right) R_{W,{ }^{*}}^{\text {nor }}\left(z^{-1}\right)$. Hence we have

$$
\mathscr{T}_{r}\left(d_{V W}(z) R_{V W}^{\text {nor }}(z)\right) \equiv d_{W, * V}\left(z^{-1}\right) R_{W, * V}^{\text {nor }}\left(z^{-1}\right) \bmod k\left[z, z^{-1}\right]^{\times} .
$$

Then the result follows from $R_{W,{ }_{V}}^{\text {nor }}\left(z^{-1}\right)=\left(R_{* V, W}^{\text {nor }}(z)\right)^{-1}$ and a well known result $\operatorname{Tr}_{r}\left(R_{V W}^{\mathrm{unv}}(z)\right)=\left(R_{* V, W}^{\text {univ }}(z)\right)^{-1}($ see $[8])$.

This proposition implies

$$
a_{\imath, j}(z) a_{\imath^{*}, j}\left(p^{*-1} z\right) \equiv \frac{d_{l, j}(z)}{d_{,, t}\left(p^{*} z^{-1}\right)} \bmod k\left[z, z^{-1}\right]^{\times} .
$$

Applying (A.8) with $* V$ instead of $V$, we have

$$
a * V, W(z) a_{* * V, W}(z) \equiv \frac{d * V, W}{d_{W, * * V}\left(z^{-1}\right)} \bmod k\left[z, z^{-1}\right]^{\times} .
$$

Using (A.5) we obtain the $q$-difference equation

$$
\frac{a_{V W}(z)}{a_{V W}\left(q^{-2(\delta, \rho)} z\right)} \equiv \frac{d_{V W}(z) d_{W V}\left(q^{2(\tilde{o}, \rho)} z^{-1}\right)}{d_{W, * V}\left(z^{-1}\right) d_{* V, W}(z)} \bmod k\left[z, z^{-1}\right] \times
$$

Write

$$
d_{\jmath \imath}(z)=\prod_{\nu}\left(z-x_{\nu}\right) \text { and } d_{,, 2}(z)=\prod_{\nu}\left(z-y_{\nu}\right) .
$$

Then by (A.6), we have

$$
d_{l j}(z)=\prod_{\nu}\left(z-\bar{x}_{\nu}^{-1}\right) \text { and } d_{i, j}(z)=\prod_{\nu}\left(z-\bar{y}_{\nu}^{-1}\right) .
$$

Then using (A.4), we can solve the $q$-difference equation(A.12),

$$
a_{t j}(z)=q^{(\pi, \pi))} \frac{\prod_{\nu}\left(p^{*} y_{\nu} z ; p^{* 2}\right)_{\infty}\left(p^{*} \overline{y_{\nu}} z ; p^{* 2}\right)_{\infty}}{\prod_{\nu}\left(x_{\nu} z ; p^{* 2}\right)_{\infty}\left(p^{* 2} \overline{x_{\nu}} z ; p^{* 2}\right)_{\infty}} .
$$

Here $\left.p^{*}=(-1)^{\prime \delta} \rho^{\prime}\right) q^{(\delta, \rho)}$ and $(z ; q)_{\infty}=\prod_{n=0}^{\infty}\left(1-q^{n} z\right)$.

We are going to determine $d_{\imath \jmath}(z)$ and $a_{\imath}(z)$ in the $A_{n-1}^{(1)}$ and $C_{n}^{(1)}$ cases.

Remark. We can see easily

$$
d_{V *, W *}(z) \equiv d_{*_{V}, * W}(z) \equiv d_{V, W}(z) .
$$


Hence

$$
a_{V^{*}, W *}(z)=a_{* V, * W}(z)=a_{V, W}(z)
$$

and

$$
d_{i *, j^{*}}(z) \equiv d_{\imath, j}(z)
$$

\section{Appendix B. $\mathbb{A}_{n-1}^{(1)}$ Case}

We shall review the fundamental representations and $R$-matrices for $A_{n-1}^{(1)}$.

B.1. Fundamental representations. The root data of $g=A_{n-1}^{(1)}$ are as follows.

$$
\begin{aligned}
& I=\{0,1, \ldots, n-1\} \\
& \left(\alpha_{i}, \alpha_{J}\right)= \begin{cases}2 & \text { if } i=j \\
-\delta(i \equiv j+1 \bmod n)-\delta(i \equiv j-1 \bmod n) & \text { otherwise }\end{cases} \\
& \delta=\alpha_{0}+\cdots+\alpha_{n-1} \text {, } \\
& c=h_{0}+\cdots+h_{n-1} \text {, } \\
& (\delta, \rho)=\left(\delta, \rho^{\vee}\right)=n+1 \text {. }
\end{aligned}
$$

Here for a statement $P$, we define $\delta(P)=1$ or -1 according that $P$ is true or false.

Hence by (1.6) the duality morphisms are given by

$$
k \stackrel{\iota}{\longrightarrow} V\left(\pi_{n-\imath}\right)_{(-q)^{n+1}} \otimes V\left(\pi_{i}\right) \text { and } V\left(\pi_{i}\right) \otimes V\left(\pi_{n-i}\right)_{(-q) n+1} \stackrel{\operatorname{tr}}{\longrightarrow} k .
$$

By [16], the vectors of the crystal base $B_{k}$ of the fundamental representation $V\left(\pi_{k}\right)\left(1 \leq_{k} \leq n\right)$ are labeled by the subsets of $\mathbb{Z} / n \mathbb{Z}=\{1, \cdots, n\}$ with exactly $k$ elements. For $0 \leq i \leq n-1$ and $K \subset \mathbb{Z} / n \mathbb{Z}$, we have

$$
\begin{aligned}
& \tilde{e}_{i}(K)= \begin{cases}(K \backslash\{i+1\}) \cup\{i\} & \text { if } i+1 \in K \text { and } i \notin K, \\
0 & \text { otherwise, }\end{cases} \\
& \tilde{f}_{\mathfrak{l}}(K)= \begin{cases}(K \backslash\{i\}) \cup\{i+1\} & \text { if } i \in K \text { and } i+1 \notin K, \\
0 & \text { otherwise. }\end{cases}
\end{aligned}
$$

In the case of the fundamental representations of $U_{q}^{\prime}\left(\widehat{\mathfrak{S l}}_{n}\right)$, all the weights are extremal. Therefore we have $e_{i} G(b)=G\left(\tilde{e}_{i} b\right)$ and $f_{i} G(b)=G\left(\tilde{f}_{i} b\right)$ for every $b$ in the crystal base. Here $G(b)$ is the corresponding global base. Hence we can and do identify its crystal bases with the corresponding global bases.

We have

$$
t_{i} K=q^{\delta(t \in K)-\delta(t+1 \in K)} K
$$

We present a lemma that is easily verified by calculation. 
Lemma B.1. For $j, k \geq 0$ such that $j+k \leq n$, there exist following non-zero $U_{q}^{\prime}\left(\widehat{\mathfrak{B l}}_{n}\right)$-linear homomorphisms.

(1) $i_{j, k}: V\left(\pi_{j+k}\right) \rightarrow V\left(\pi_{j}\right)_{(-q) k} \otimes V\left(\pi_{k}\right)_{(-q)-}$ given by

$$
i_{j, k}(M)=\sum_{\substack{\sharp J=j, \# K=k \\ M=J \cup K, J \cap K=\emptyset}}(-q)^{\psi(J, K)} J \otimes K .
$$

Here $\psi(J, K)=\#\{(\nu, \mu) \in J \times K ; \nu>\mu\}$.

(2) $p_{j, k}: V\left(\pi_{j}\right)_{(-q)^{-k}} \otimes V\left(\pi_{k}\right)_{(-q)}, \rightarrow V\left(\pi_{\jmath+k}\right)$

given by

$$
p_{j, k}(J \otimes K)= \begin{cases}(-q)^{\phi(J, K)}(J \cup K) & \text { if } J \cap K=\emptyset \\ 0 & \text { if } J \cap K \neq \emptyset .\end{cases}
$$

Here $V\left(\pi_{0}\right)$ and $V\left(\pi_{n}\right)$ are understood to be the trivial representation.

B.2. $\boldsymbol{R}$-matrices. We shall recall the result of Date-Okado [5].

Proposition B.2([5]). For $k, l \in I_{0}$

$$
d_{k l}(z)=\prod_{\nu=1}^{\min (k, l, n-k, n-l)}\left(z-(-q)^{2 \nu+|k-l|}\right) .
$$

The univeral $R$-matrices can be easily obtained by (A.13) and (B.1).

Proposition $\mathbb{B} .3([5])$. For $k, l \in I_{0}=\{1, \cdots, n-1\}$, we hav'e

$$
a_{k l}(z)=q^{\min (k, l)-k l / n} \frac{\left((-q)^{|k-l|} z ; q^{2 n}\right)_{\infty}\left((-q)^{2 n-|k-l|} z: q^{2 n}\right)_{\infty}}{\left((-q)^{k+l} z ; q^{2 n}\right)_{\infty}\left((-q)^{2 n-k-l} z ; q^{2 n}\right)_{\infty}} .
$$

\section{Appendix $\mathbb{C} . C_{n}^{(1)}$ Case}

C.1. Fundamental representations. The Dynkin diagram of $C_{n}^{(1)}$ is

$$
\bigcirc_{-2 \varepsilon_{1}}^{0} \Rightarrow \bigcirc_{\varepsilon_{1}-\varepsilon_{2}}^{1}-\bigcirc_{\varepsilon_{2}-\varepsilon_{3}}^{2}-\cdots-\bigcirc_{\varepsilon n-1-\varepsilon_{n}}^{n-1} \Leftarrow \bigcirc_{2 \varepsilon_{n}}^{n}
$$

Here $\left(\varepsilon_{\imath}\right)_{\imath=1, \ldots, n}$ is an orthogonal basis of $\mathrm{t}_{\mathrm{cl}}^{* 0}$ such that $\left(\varepsilon_{\imath}, \varepsilon_{\imath}\right)=1 / 2$. We have

$$
\begin{aligned}
q_{\imath} & = \begin{cases}q & \text { if } i=0 \text { or } n \\
q^{1 / 2} & \text { if } 1 \leq i<n,\end{cases} \\
\delta & =\alpha_{0}+2\left(\alpha_{1}+\cdots \alpha_{n-1}\right)+\alpha_{n}, \\
c & =h_{0}+h_{1}+\cdots+h_{n}, \\
(\delta, \rho) & =n+1, \\
\left\langle\rho^{\vee}, \delta\right\rangle & =2 n,
\end{aligned}
$$




$$
\pi_{i}=\Lambda_{i}-\Lambda_{0}=\varepsilon_{1}+\cdots+\varepsilon_{\imath} .
$$

We set $q_{s}=q^{1 / 2}$. Hence by $(1.6)$ the duality morphisms are given by

$$
k \stackrel{c}{\longrightarrow} V\left(\pi_{\imath}\right)_{q_{S}^{2(n+1)}} \otimes V\left(\pi_{l}\right) \text { and } V\left(\pi_{l}\right) \otimes V\left(\pi_{\imath}\right)_{q_{s}^{2(n+1)}} \stackrel{\operatorname{tr}}{\longrightarrow} k .
$$

We review the crystal base $\left(L_{k}, B_{k}\right)$ of the fundamental representation $V\left(\pi_{k}\right)$ (1 $\left.\leq_{k} \leq_{n}\right)$ of $U_{q}^{\prime}\left(C_{n}^{(1)}\right)$. Recall that $V\left(\pi_{k}\right)$ is as a $U_{q}\left(C_{n}\right)$-module isomorphic to the $k$-th fundamental representation of $U_{q}\left(C_{n}\right)$. Hence by [17], $B_{k}$ is labeled by

$$
\left\{\left(m_{t}\right)_{i=1}^{k} ; m_{1} \prec \cdots \prec m_{k}, m_{t} \in\left\{1, \ldots, n, \bar{n}_{, \ldots .,}, \overline{1}_{,}\right\}, i+(k-j+1) \leq m_{\imath} \text { if } m_{t}=\bar{m}_{j}(i<j)\right\},
$$

where the ordering on $\{1, \ldots, n, \bar{n}, \ldots, \overrightarrow{1}\}$ is defined by

$$
1 \prec \cdots \prec n \prec \bar{n}<\cdots<\overline{1} \text {. }
$$

On $B_{k}$ the actions of $\tilde{f}_{t}$ and ${\widetilde{e_{i}}}_{i}$ with $0 \leq_{i} \leq_{n}$ are defined as follows. As for $i \neq 0$, write $i$ and $\overline{i+1}$ as,$+ i+1$ and $\bar{i}$ as - , and others as 0 . Then first ignore 0 and next ignore +- . Then $\tilde{f}_{i} b$ is obtained by replacing the leftmost + with - and $\tilde{e}_{t} b$ is obtained by replacing the rightmost - with + .

Lemmina $\mathbb{C}_{0} \mathbb{1}$. If $b$ is of the form $\left(1, a_{1}, \ldots, a_{k-1}\right)$, then $\tilde{e}_{0} b=\left(a_{1}, \ldots, a_{k-1}, \overline{1}\right)$. Otherwise $\widetilde{e}_{0} b=0$. If $b$ is of the form $\left(a_{1}, \ldots, a_{k-1}, \overline{1}\right)$, then $\widetilde{f}_{0} b=\left(1, a_{1}, \ldots, a_{k-1}\right)$. Otherwise $\tilde{f}_{0} b=0$.

Proof. It is easy to check that $B_{k}$ is a regular crystal with this definition of ${\widetilde{e_{0}}}_{\text {and }} \widetilde{f}_{0}$. Set $J=\{1,2, \cdots, n-1\} \subset I$. Then $B_{k}$ decomposes. as a crystal over $g_{J} \simeq$ $A_{n-1}$. into irreducible components with multiplicity 1 . Hence there is a unique way to draw 0-arrows on the crystal $B_{k}$ over $C_{n}$.

The following proposition can be checked by a direct calculation.

Proposition $\mathbb{C}$.2. For $\mu . \nu$ with $\mu+\nu \leq n$, there exist follouing non-zero $U_{q}^{\prime}\left(C_{n}^{(1)}\right)$-linear maps:

$$
\begin{aligned}
& i_{\mu \nu}: V\left(\pi_{\mu+\nu}\right) \rightarrow V\left(\pi_{\mu}\right)_{\left(-q_{s}\right) \nu} \otimes V\left(\pi_{\nu}\right)_{(-q)^{-\mu},} \\
& p_{\mu \nu}: V\left(\pi_{\mu}\right)_{\left(-q_{s}\right)^{-\nu}} \otimes V\left(\pi_{\nu}\right)_{\left(-q_{s}\right)^{\mu}} \rightarrow V\left(\pi_{\mu+\nu}\right) .
\end{aligned}
$$

C.2. Normalized $\mathbb{R}$-matrices. Let us calculate $R$-matrices between a fundamental representation and the vector representation of $U_{q}^{\prime}\left(C_{n}^{(1)}\right)$. First recall that we have the following decomposition as $U_{q}\left(C_{n}\right)$-modules;

$$
V\left(\pi_{k}\right) \otimes V\left(\pi_{1}\right)=V\left(\pi_{k}+\pi_{1}\right) \oplus V\left(\pi_{k+1}\right) \oplus V\left(\pi_{k-1}\right) .
$$

Here $V\left(\pi_{0}\right)$ is understood to be the trivial representation and $V\left(\pi_{n+1}\right)$ to be 0 . Therefore the $R$-matrix $R_{k 1}^{\text {nor }}(x, y): V\left(\pi_{k}\right)_{x} \otimes V\left(\pi_{1}\right)_{y} \rightarrow V\left(\pi_{1}\right)_{y} \otimes V\left(\pi_{k}\right)_{x}$ can bc 
written as $R_{k 1}^{\text {nor }}(x, y)=\mathrm{P}_{\pi_{k}+\pi_{1}} \oplus \gamma_{1}(y / x) \mathrm{P}_{\pi_{k+1}} \oplus \gamma_{2}(y / x) \mathrm{P}_{\pi k-1}$, where $P_{\pi}$ is a $U_{q}\left(C_{n}\right)$-linear projection from $V\left(\pi_{k}\right) \otimes V\left(\pi_{1}\right)$ to $V(\pi)$ in $V\left(\pi_{1}\right) \otimes V\left(\pi_{k}\right)$ with $\pi$ $=\pi_{k}+\pi_{1}, \pi_{k+1}$ or $\pi_{k-1}$.

Let $u_{i}$ and $u_{i}^{\prime}(i=0,1,2)$ be highest-weight vectors in the $U_{q}\left(C_{n}\right)$-modules $V\left(\pi_{k}\right) \otimes V\left(\pi_{1}\right)$ and $V\left(\pi_{1}\right) \otimes V\left(\pi_{k}\right)$ with highest weights $\pi_{k}+\pi_{1}(i=0), \pi_{k+1}(i=$ $1), \pi_{k-1}(i=2)$. Remark that if $k=n$ we ignore $\pi_{k+1}, u_{1}, u_{1}^{\prime}$ and $\gamma_{1}(y / x)$. We set $Q_{1}=f_{0} f_{1} \cdots f_{n-1} f_{n} f_{n-1} \cdots f_{k+1}$ and $Q_{2}=f_{0} f_{1} \cdots f_{k-1}$. Then $Q_{i} u_{t}$ is proportional to $u_{0}$ because its weight is $\pi_{k}+\pi_{1}$. Let us first determine $\gamma_{1}$, assuming that $k \neq n$.

The following lemma is by direct calculation and we leave it to the reader. In the sequel $G$ means the lower global base (cf. $[12,13]$ ).

Lemma C.3. Let $b$ be an element of $V\left(\pi_{k}\right) \otimes V\left(\pi_{1}\right)$ which is a tensor product of two lower global bases of $V\left(\pi_{k}\right)$ and $V\left(\pi_{1}\right)$ and has the weight $\pi_{k+1}$. Then $Q_{1} b \neq 0$ if and only if $b=b_{1}:=G(1, \ldots, k) \otimes G(k+1)$ or $b=b_{2}:=G(2, \ldots, k+1)$ $\otimes G(1)$. Mororver $Q_{1} b_{1}=q^{-1} y^{-1} u_{0}$ and $Q_{1} b_{2}=x^{-1} u_{0}$, where we sct $u_{0}=G(1 \ldots, k) \otimes$ $G(1)$.

Lemma C.4. If we write $u_{1}=b_{1}+\sum_{b \neq b_{1}} a_{b} b$, where $b$ runs over the set of tensor products of two lower global bases, then $a_{b 2}=\left(-q_{s}\right)^{k}$.

Proof. There are relations

$$
\begin{array}{r}
e_{2}\left(G(1, \ldots, i \hat{+} 1, \ldots, k+1) \otimes G(i+1)-q_{s} G(1, \ldots, \hat{i}, \ldots, k+1) \otimes G(i)\right)=0 \\
\text { for } 1 \leq i \leq k .
\end{array}
$$

It follows that $a_{b_{2}}=\left(-q_{s}\right)^{k}$.

By these lemmas we have $Q_{1} \imath_{1}=\left(q_{s}^{-1} y^{-1}+\left(-q_{s}\right)^{k} x^{-1}\right) u_{0}$ in $V\left(\pi_{k}\right) \otimes$ $I^{r}\left(\pi_{1}\right)$.

Similary we obtain the following two lemmas.

Lemma C.5. Let $b$ be an element of $V\left(\pi_{1}\right) \otimes V\left(\pi_{k}\right)$ which is a tensor product of tuo lower global bases of $r\left(\pi_{1}\right)$ and $V\left(\pi_{k}\right)$ and has the weight $\pi_{k+1}$ Then $Q_{1} b \neq 0$ if and only if $b=b_{1}^{\prime}:=G(1) \otimes G(2, \ldots, k+1)$ or $b=b_{2}^{\prime}:=G(k+1) \otimes$ $G(1 \ldots k)$. Moreover $Q_{1} b_{1}^{\prime}=q_{s}^{-1} x^{-1} u_{0}^{\prime}$ and $Q_{1} b_{2}^{\prime}=y^{-1} u_{0}^{\prime}$, where we set $u_{0}^{\prime}=G(1) \otimes$ $G(1, \ldots, k)$.

Lemma C.6. If we write $u_{1}^{\prime}=b_{1}^{\prime}+\sum_{b \neq b 1} a_{b} b$, where $b$ runs over the set of tensor products of two lower global bases, then $a_{b 2}=\left(-q_{s}\right)^{k}$.

By these lemmas we have in $V\left(\pi_{1}\right) \otimes V^{r}\left(\pi_{k}\right)$

$$
Q_{1} u_{1}^{\prime}=\left(q_{s}^{-1} x^{-1}+\left(-q_{s}\right)^{h} y^{-1}\right) u_{0}^{\prime} .
$$

Therefore we have 


$$
\gamma_{1}=\frac{x-\left(-q_{s}\right)^{k+1} y}{y-\left(-q_{s}\right)^{k+1} x}
$$

Next let us determine $\gamma_{2}$. For brevity, we assume that $k \neq n$ in the following four lemmas.

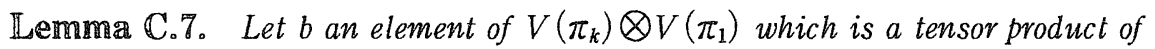
two lower global bases of $V\left(\pi_{k}\right)$ and $V\left(\pi_{1}\right)$ and has the weight $\pi_{k-1}$. Then $Q_{2} b \neq 0$ if and only if $b=b_{3}:=G(1, \ldots, k) \otimes G(\bar{k})$ or $b=b_{4}:=G(2, \ldots, k, \bar{k}) \otimes G(1)$. Moreover $Q_{2} b_{3}=q_{s}^{-2} y^{-1} u_{0}$ and $Q_{2} b_{4}=x^{-1}[2]_{1} u_{0}$, where we set $u_{0}=G(1, \ldots, k) \otimes G(1)$.

This lemma is by direct calculation and we leave it to the reader.

Lemma $\mathbb{C}$.8. If we write $u_{2}=b_{3}+\sum_{b \neq b_{3}} a_{b} b$, where $b$ runs over the set of tensor products of two lower global bases, then $a_{b_{4}}=-\left(-q_{s}\right)^{2 n-k+1} /[2]_{k-1}$.

Proof. There are relations.

$$
\begin{aligned}
& e_{i}\left(G(1, \ldots, k-1, i) \otimes G(\bar{i})-q_{s} G(1, \ldots, k-1, i+1) \otimes G(\overline{i+1})\right)=0 \\
& \text { for } i=k, \ldots, n-1 \text {, } \\
& e_{n}\left(G(1, \ldots, k-1, n) \otimes G(\vec{n})-q_{s}^{2} G(1, \ldots, k-1, \bar{n}) \otimes G(n)\right)=0 \text {, } \\
& e_{i}\left(G(1, \ldots, k-1, \overline{i+1}) \otimes G(i+1)-q_{s} G(1, \ldots, k-1, \bar{i}) \otimes G(i)\right)=0 \\
& \text { for } i=k, \ldots, n-1 \text {, } \\
& e_{k-1}\left([2]_{k-1} G(1, \ldots, k-1, \bar{k}) \otimes G(k)-q_{s} G(1, \ldots, k-2, k, \bar{k}) \otimes G(k-1)\right)=0 \text {, } \\
& e_{l}\left(G(1, \ldots, i \mp 1, \ldots, k, \bar{k}) \otimes G(i+1)-q_{s} G(1, \ldots, \bar{i}, \ldots, k, \bar{k}) \otimes G(i)\right)=0 \\
& \text { for } i=1, \ldots, k-2 \text {. }
\end{aligned}
$$

It follows that $a_{b 4}=-\left(-q_{s}\right)^{2 n-k+1} /[2]_{k-1}$.

By these lemmas we have in $V\left(\pi_{k}\right) \otimes V\left(\pi_{1}\right)$

$$
Q_{2} u_{2}=\left(q_{s}^{-2} y^{-1}-\left(-q_{s}\right)^{2 n-k+1} x^{-1}\right) u_{0}
$$

Similarly we obtain the following two lemmas for $V\left(\pi_{1}\right) \otimes V\left(\pi_{k}\right)$.

Lemma $\mathbb{C}$.9. Let $b$ be an element of $V\left(\pi_{1}\right) \otimes V\left(\pi_{k}\right)$ which is a tensor product of two lower global bases of $V\left(\pi_{1}\right)$ and $V\left(\pi_{h}\right)$ and has the weight $\pi_{k-1}$. Then $Q_{2} b \neq 0$ if and only if $b=b_{3}^{\prime}:=G(1) \otimes G(2, \ldots, k, \bar{k})$ or $b=b_{4}^{\prime}:=G(\bar{k}) \otimes G(1, \ldots$, k). Moreover $Q_{2} b_{3}^{\prime}=q_{s}^{-1} x^{-1}[2]_{1} u_{0}^{\prime}$ and $Q_{2} b_{4}^{\prime}=y^{-1} u_{0}^{\prime}$, where we set $u_{0}^{\prime}=G(1) \otimes G(1, \ldots$, k).

Lemma $\mathbb{C}$.10. If we write $u_{2}^{\prime}=b_{3}^{\prime}+\sum_{b \neq b 3} a_{b} b$, where $b$ mins over the set of tensor products of two lower global bases, then $a_{b 4}=\left(-q_{s}\right)^{2 n-k+2}[2]_{k-1}$.

By these lemmas we have in $V\left(\pi_{1}\right) \otimes V\left(\pi_{k}\right)$

$$
Q_{2} u_{2}^{\prime}=\left(q_{s}^{-1} x^{-1}+\left(-q_{s}\right)^{2 n-k+2} y^{-1}\right)\left(q_{s}+q_{s}^{-1}\right) u_{0}^{\prime} .
$$


Therefore up to a multiple of an element of $k$ we have

$$
\gamma_{2}=\frac{x-\left(-q_{s}\right)^{2 n-k+3} y}{y-\left(-q_{s}\right)^{2 n-k+3} x}
$$

It is easy to check that this expression for $\gamma_{2}$ still holds even if $k=n$. So we obtain the following result.

Theorem C.11. The normalized $R$-matrix is given by

$$
R_{k 1}^{\text {nor }}(z)= \begin{cases}\mathrm{P}_{\pi_{k+\pi}}+\frac{1-\left(-q_{s}\right)^{k+1} z}{z-\left(-q_{s}\right)^{k+1}} \mathrm{P}_{\pi_{k+1}}+\frac{1-\left(-q_{s}\right)^{2 n-k+3} z}{z-\left(-q_{s}\right)^{2 n-k+3}} \mathrm{P}_{\pi_{k-1}} & \text { if } 1 \leq k<n, \\ \mathrm{P}_{\pi_{k}+\pi_{1}}+\frac{1-\left(-q_{s}\right)^{n+3} z}{z-\left(-q_{s}\right)^{n+3}} \mathrm{P}_{\pi_{k-1}} & \text { if } k=n .\end{cases}
$$

Hence we have

(C.9) $\quad d_{1, k}(z)=d_{k 1}(z)= \begin{cases}\left(z-\left(-q_{s}\right)^{k+1}\right)\left(z-\left(-q_{s}\right)^{2 n+3-k}\right) & \text { if } 1 \leq k<n, \\ z-\left(-q_{s}\right)^{n+3} & \text { if } k=n .\end{cases}$

We give the explicit form of $R$-matrix for the vector representaion.

Proposition C.12. For $b_{1}, b_{2} \in B\left(\pi_{1}\right)$ we have

$$
R_{11}^{\text {nor }}(z)\left(b_{1} \otimes b_{2}\right)= \begin{cases}b_{1} \otimes b_{2} & \text { if } b_{1}=b_{2}, \\ \frac{\left(1-q_{s}^{2}\right) z^{\delta\left(b_{2}<b_{1}\right)}}{z-q_{s}^{2}} b_{1} \otimes b_{2}+\frac{q_{s}(z-1)}{z-q_{s}^{2}} b_{2} \otimes b_{1} & \text { if } b_{1} \neq b_{2}, \overline{b_{2}} .\end{cases}
$$

For $1 \leq a \leq n$ we have

$$
\begin{aligned}
& R_{11}^{\mathrm{nor}}(z)(a \otimes \bar{a})=\frac{1-q_{s}^{2}}{z-q_{s}^{2}} a \otimes \bar{a}+\sum_{k=1}^{n} \frac{\left(-q_{s}\right)^{a+k}\left(1-q_{s}^{2}\right)(z-1)}{\left(z-q_{s}^{2}\right)\left(z-\left(-q_{s}\right)^{2 n+2}\right)} k \otimes \bar{k} \\
&-\sum_{k>a} \frac{\left(-q_{s}\right)^{2 n+a-k+2}\left(1-q_{s}^{2}\right)(z-1)}{\left(z-q_{s}^{2}\right)\left(z-\left(-q_{s}\right)^{2 n+2}\right)} \bar{k} \otimes k+\frac{q_{s}^{2}(z-1)\left(z-\left(-q_{s}\right)^{2 n}\right)}{\left(z-q_{s}^{2}\right)\left(z-\left(-q_{s}\right)^{2 n+2}\right)} \bar{a} \otimes a \\
& \quad-\sum_{k<a} \frac{\left(-q_{s}\right)^{a-k}\left(1-q_{s}^{2}\right) z(z-1)}{\left(z-q_{s}^{2}\right)\left(z-\left(-q_{s}\right)^{2 n+2}\right)} \bar{k} \otimes k, \\
&\left.\left.R_{11}^{\text {nor }}(z)(\bar{a} \otimes a)=-\sum_{k<a} \frac{\left(-q_{s}\right)^{2 n-a+k+2}}{\left(z-q_{s}^{2}\right)\left(z-q_{s}^{2}\right)(z-1)} k \bar{q}_{s}\right)^{2 n+2}\right) \\
&+ \frac{q_{s}^{2}(z-1)\left(z-\left(-q_{s}\right)^{2 n}\right)}{\left(z-q_{s}^{2}\right)\left(z-\left(-q_{s}\right)^{2 n+2}\right)} a \otimes \bar{a}-\sum_{k>a} \frac{\left(-q_{s}\right)^{k-a}\left(1-q_{s}^{2}\right) z(z-1)}{\left(z-q_{s}^{2}\right)\left(z-\left(-q_{s}\right)^{2 n+2}\right)} k \otimes \bar{k} \\
&+ \sum_{k=1}^{n} \frac{\left(-q_{s}\right)^{2 n-a-k+2}\left(1-q_{s}^{2}\right) z(z-1)}{\left(z-q_{s}^{2}\right)\left(z-\left(-q_{s}\right)^{2 n+2}\right)} \bar{k} \otimes k+\frac{\left(1-q_{s}^{2}\right) z}{z-q_{s}^{2}} \bar{a} \otimes a .
\end{aligned}
$$


The general $d_{i j}$ with $i, j \neq 1$ will be calculated at the end of this section with the aid of the universal $R$-matrices.

$\mathbb{C}$.ङ. Universal $\mathbb{R}$-matrices. We shall calculate the universal $R$-matrices. By (A.13) and (C.9), we have

$$
a_{1 k}(z)=a_{k 1}(z)=q_{s} \frac{\{k-1\}\{2 n+1-k\}\{2 n+3+k\}\{4 n+5-k\}}{\{k+1\}\{2 n+3-k\}\{2 n+1+k\}\{4 n+3-k\}} .
$$

Here we employed the notation

$$
\{m\}=\left(\left(-q_{s}\right)^{m} z ; q_{s}^{4 n+4}\right)_{\infty} .
$$

Now we shall calculate $a_{k l}(z)$ for $l \leq k$. Consider the commutative diagram

$$
\begin{array}{ll}
V\left(\pi_{k}\right) \otimes V\left(\pi_{l-1}\right)_{\substack{\left(-q_{s}\right)^{-1} z \\
f \downarrow}} \otimes V\left(\pi_{1}\right)_{\left(-q_{s}\right)^{l-1} z} & \stackrel{\psi}{\longrightarrow} V\left(\pi_{k}\right) \otimes V\left(\pi_{l}\right)_{z} \\
V\left(\pi_{l-1}\right)_{\left(-q_{s}\right)^{-1 z}} \otimes V\left(\pi_{k}\right) \otimes V\left(\pi_{1}\right)_{\left(q_{s}\right)^{l-1 z}} & \\
I^{\prime}\left(\pi_{l-1}\right)_{\left(-q_{S}\right)^{-1} z} \otimes V\left(\pi_{1}\right)_{\left(-q_{s}\right)^{l-1_{z}}} \otimes V\left(\pi_{k}\right) & \underset{\psi^{\prime}}{\longrightarrow} V\left(\pi_{l}\right)_{z} \otimes V\left(\pi_{k}\right)^{\longrightarrow} .
\end{array}
$$

Here

$$
\begin{aligned}
& \phi=V\left(\pi_{k}\right) \otimes\left(p_{l-1,1}\right)_{z}, \phi^{\prime}=\left(p_{l-1,1}\right)_{z} \otimes V\left(\pi_{k}\right), \\
& f=R_{k, l-1}^{\mathrm{univ}}\left(\left(-q_{s}\right)^{-1} z\right) \otimes V\left(\pi_{1}\right)_{\left(-q_{s}\right)^{l-1} z}, \\
& g=V\left(\pi_{l-1}\right)_{\left(-q_{s}\right)^{-1} z} \otimes R_{k 1}^{\text {univ }}\left(\left(-q_{s}\right)^{l-1} z\right) \text { and } h=R_{k l}^{\text {univ }}(z) .
\end{aligned}
$$

We have

$$
\begin{gathered}
p_{l-1,1}(G(1, \ldots, l-1) \otimes G(l))=G(1, \ldots, l), \\
R_{k, 1}^{\text {nor }}(z)(G(1, \ldots, k) \otimes G(l)=G(l) \otimes G(1, \ldots, k) .
\end{gathered}
$$

Chasing the vector $G(1 \ldots ., k) \otimes G(1, \ldots ., l-1) \otimes G(l)$ of $V\left(\pi_{k}\right) \otimes V\left(\pi_{l-1}\right)_{\left(-q_{s}\right)^{-1} z} \otimes$ $V\left(\pi_{1}\right)_{\left(-q_{s}\right)^{t-1 z}}$ in the diagram C.12, we obtain the recurrence relation

$$
a_{k l}(z)=a_{k, l-1}\left(\left(-q_{s}\right)^{-1} z\right) a_{k, 1}\left(\left(-q_{s}\right)^{l-1} z\right) .
$$

Solving this, and noticing $a_{k l}=a_{l k}$, we obtain the following result.

Proposition $\mathbb{C} .13$. For $k, l \in I_{0}=\{1, \ldots, n\}$, we have

$$
a_{k l}(z)=q_{s}^{\min (k, l)} \frac{\{|k-l|\}\{2 n+2-k-l\}\{2 n+2+k+l\}\{4 n+4-|k-l|\}}{\{k+l\}\{2 n+2-k+l\}\{2 n+2+k-l\}\{4 n+4-k-l\}} .
$$

Here we used the notation $\{m\}=\left(\left(-q_{s}\right)^{m} z ; q_{s}^{4 n+4}\right)_{\infty}$.

C.4. Denominators of normalized $\mathbb{R}$-matrices. In this subsection we shall prove

Proposition $\mathbb{C}$.14. For $1 \leq k, l \leq n$, we have 
(C. 13) $d_{k l}(z)=\prod_{i=1}^{\min (k, l, n-k, n-l)}\left(z-\left(-q_{s}\right)^{|k-l|+2 \imath}\right) \prod_{i=1}^{\min (k, l)}\left(z-\left(-q_{s}\right)^{2 n+2-k-l+2 i}\right)$.

This is already proved in the case $l=1$. The case $k=l=n$ is proved in $[16$, Proposition 4.2.6]. We shall prove this proposition by reduction to those cases. Let $D_{k l}(z)$ be the right hand side of (C.13).

By (A.6), we may assume that $k \geq l$. First let us show that $d_{k l}(z)$ is a multiple of $D_{k l}(z)$. In order to see this, by using Corollary 2.4 , it is enough to show that $V\left(\pi_{k}\right) \otimes V\left(\pi_{l}\right)_{a}$ is reducible for any root $a$ of $D_{k l}(z)$. For $1 \leq i \leq n-k$, $l$, we have

$$
\begin{aligned}
& V\left(\pi_{k}\right) \otimes V\left(\pi_{l}\right)_{\left(-q_{s}\right)^{k-l+2 t}} \\
& V^{\prime}\left(\pi_{k}\right) \otimes\left(i_{1, l-1}\right)_{\left(-q_{s}\right) k-1+2 !} \downarrow \\
& V\left(\pi_{k}\right) \otimes V\left(\pi_{l}\right)_{\left(-q_{S}\right)^{k+\imath}} \otimes V\left(\pi_{l-\imath}\right)_{\left(-q_{S}\right)^{k-l+\imath}} \\
& \left(p_{k t}\right)_{\left(-q_{s}\right)} \otimes V\left(\pi_{l-\xi}\right)_{(-q s) k^{-l+1}} \downarrow \\
& V\left(\pi_{k+i}\right)_{\left(-q_{S}\right)^{t}} \otimes V\left(\pi_{l-\imath}\right)_{\left(-q_{S}\right)^{k-l+i}}
\end{aligned}
$$

Here $V\left(\pi_{0}\right)$ is understood to be the trivial representation. Then one can easily see that the composition is not zero but $u_{k} \otimes u_{l}$ is sent to zero. Hence $V\left(\pi_{k}\right) \otimes$ $V\left(\pi_{l}\right)_{(-q s)^{k-l+2 t}}$ is reducible. Similarly for $1 \leq i \leq l$, let us consider

$$
\begin{aligned}
& \left.V\left(\pi_{k}\right) \otimes V\left(\pi_{l}\right)_{(-q s)}\right)^{2 n+2-k-l+2 t} \\
& \imath_{k-1} \otimes\left(l_{t} \mid-t\right)_{\left(-q_{s}\right) 2 n+2-k-1+2 t} \downarrow \\
& V\left(\pi_{k-\imath}\right)_{\left(-q_{S}\right)^{t}} \otimes V^{r}\left(\pi_{\imath}\right)_{\left(-q_{S}\right)^{t-k}} \otimes V\left(\pi_{l}\right)_{\left(-q_{S}\right)^{2 n+2-k+t}} \otimes V\left(\pi_{l-t}\right)_{\left(-q_{S}\right)^{2 n+2-k-l+t}} \\
& V^{\prime}\left(\pi_{k-t}\right)_{(-q s)} \otimes \operatorname{tr} \otimes V\left(\pi_{l-t}\right)_{(-q s) 32 n+2-k-1+t} \downarrow \\
& V\left(\pi_{k-1}\right)_{\left(-q_{S}\right)} \otimes V\left(\pi_{l-1}\right)_{\left(-q_{S}\right)^{2 n+2-k-l+1}}
\end{aligned}
$$

In this case also, the composition is not zero but $u_{k} \otimes u_{l}$ is sent to zero. Hence

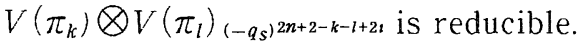

By (A.10), we have

$$
a_{k l}(z) a_{k l}\left(\left(-q_{s}\right)^{-(2 n+2)} z\right) \equiv \frac{d_{k l}(z)}{d_{k l}\left(\left(-q_{s}\right)^{2 n+2} z^{-1}\right)} \quad \bmod k\left[z, z^{-1}\right]^{\times} .
$$

Hence we obtain

$$
d_{k l}(z)=D_{k l}(z) \phi_{k l}(z)
$$

for a polynomial $\phi_{k l}(z)$ satisfying

$$
\phi_{k l}(z) \equiv \phi_{k l}\left(\left(-q_{s}\right)^{2 n+2} z^{-1}\right) \quad \bmod k\left[z, z^{-1}\right]^{\times} .
$$

Now we shall use the following lemma.

Lemma C.15. Let $V^{\prime}, V^{\prime \prime}, V$ and $W$ be irreducible $U_{q}^{\prime}(\mathrm{g})$-modules. Assume that there is a surjective morphism $V^{\prime} \otimes V^{\prime \prime} \rightarrow V$. Then 


$$
\frac{d_{W, V^{\prime}}(z) d_{W, V^{\prime}}(z) a_{W, V}(z)}{d_{W, V}(z) a_{W, V^{\prime}}(z) a_{W, V^{\prime \prime}}(z)} \text { and } \frac{d_{V^{\prime}, W}(z) d_{V^{\prime \prime}, W}(z) a_{V, W}(z)}{d_{V, W}(z) a_{V^{\prime}, W}(z) a_{V^{\prime \prime}, W}(z)}
$$

are in $k\left[z, z^{-1}\right]$.

Proof. In a commutative diagram

$$
\begin{aligned}
& W \otimes V_{z}^{\prime} \otimes V_{z}^{\prime \prime} \longrightarrow W \otimes V_{z} \\
& R^{\prime}(z) \otimes V_{z}^{\prime \prime} \downarrow \\
& V_{z}^{\prime} \otimes W \otimes V_{z}^{\prime \prime} \\
& V_{z}^{\prime} \otimes R^{\prime \prime}(z) \downarrow \\
& V_{z}^{\prime} \otimes V_{z}^{\prime \prime} \otimes W \longrightarrow{ }^{R}(z) \downarrow \\
&
\end{aligned}
$$

if $R^{\prime}(z)$ and $R^{\prime \prime}(z)$ do not have poles, then so is $R(z)$. To see the first assertion, it is enough to apply this to $R^{\prime}(z)=d_{W, V^{\prime}}(z) R_{W, V^{\prime}}^{\text {nor }}(z), R^{\prime \prime}(z)=d_{W, V^{\prime \prime}}(z) R_{W, V^{\prime \prime}}^{\text {nor }}(z)$ and

$$
R(z)=\frac{d_{W, V^{\prime}}(z) d_{W, V^{\prime \prime}}(z) a_{W, V}(z)}{a_{W, V^{\prime}}(z) a_{W, V^{\prime \prime}}(z)} R_{W, V}^{\text {nor }}(z) .
$$

The second assertion can be proved similarly.

We shall prove $\phi_{k l}(z) \equiv 1 \bmod k\left[z, z^{-1}\right]^{\times}$.

Case $k+l \leq n$. We prove this by the induction on $l$. If $l=1$, it is already proved. If $l>1$ then applying the lemma above to $V\left(\pi_{l-1}\right)_{\left(-q_{s}\right)^{-1}} \otimes V\left(\pi_{1}\right)_{\left(-q_{s}\right)^{l-1}} \longrightarrow$ $V\left(\pi_{l}\right)$. we have

$$
\frac{d_{k, l-1}\left(\left(-q_{s}\right)^{-1} z\right) d_{k, 1}\left(\left(-q_{s}\right)^{l-1} z\right) a_{k, l}(z)}{d_{k, l}(z) a_{k, l-1}\left(\left(-q_{s}\right)^{-1} z\right) a_{k, 1}\left(\left(-q_{s}\right)^{l-1} z\right)} \in k\left[z, z^{-1}\right] .
$$

Since

$$
\frac{D_{k, l-1}\left(\left(-q_{s}\right)^{-1} z\right) d_{k, 1}\left(\left(-q_{s}\right)^{l-1} z\right) a_{k, l}(z)}{D_{k, l}(z) a_{k, l-1}\left(\left(-q_{s}\right)^{-1} z\right) a_{k, 1}\left(\left(-q_{s}\right)^{l-1} z\right)} \equiv 1
$$

$\phi_{k, l-1}(z) \equiv 1$ implies $\phi_{k l}(z) \equiv 1$.

Case $k+l>n$. We shall first reduce the assertion to the $k=n$ case. For $k$ $<n$ consider a surjection

$$
V\left(\pi_{k+1}\right)_{\left(-q_{s}\right)^{-1}} \otimes V\left(\pi_{1}\right)_{\left(-q_{s}\right)^{2 n+1-k}} \rightarrow V\left(\pi_{k}\right)
$$

given by the composition

$$
\begin{aligned}
V\left(\pi_{k+1}\right)_{(-q s)^{-1}} \otimes V\left(\pi_{1}\right)_{(-q s) 2 n+1-k} \longrightarrow V\left(\pi_{k}\right) \otimes V\left(\pi_{1}\right)_{(-q s)^{-1-k}} \otimes V\left(\pi_{1}\right)_{(-q s)^{2 n+1-k}} & \rightarrow V\left(\pi_{k}\right) .
\end{aligned}
$$

We have

$$
\frac{D_{k+1, l}\left(\left(-q_{s}\right) z\right) d_{1, l}\left(\left(-q_{s}\right)^{k-2 n-1} z\right) a_{k l}(z)}{D_{k l}(z) a_{k+1, l}\left(\left(-q_{s}\right) z\right) a_{1, l}\left(\left(-q_{s}\right)^{k-2 n-1} z\right)} \equiv z-\left(-q_{s}\right)^{4 n+4-k-l} .
$$


Hence $\phi_{k+1, l}(z) \equiv 1$ implies that $\phi_{k l}(z)$ is a divisor of $z-\left(-q_{s}\right)^{4 n+4-k-l}$. Then (C.15) implies that $\phi_{k l}(z) \equiv 1$. Hence, the descending induction on $k$ reduces the problem to the $k=n$ case. We have

$$
\frac{D_{k, l-1}\left(\left(-q_{s}\right)^{-1} z\right) d_{k, l}\left(\left(-q_{s}\right)^{l-1} z\right) a_{k, l}(z)}{D_{k, l}(z) a_{k, l-1}\left(\left(-q_{s}\right)^{-1} z\right) a_{k, 1}\left(\left(-q_{s}\right)^{l-1} z\right)} \equiv z-\left(-q_{s}\right)^{2 n+2-k+l} .
$$

Hence by the similar argument to $k+l \leq n$ case, $\phi_{k, l-1}(z) \equiv 1$ implies that $\phi_{k l}(z)$ is a divisor of $z-\left(-q_{s}\right)^{2 n+2-k+l}$. Hence if $l \neq k=n$ then we can reduce the $l$ case to the $l-1$ case. This completes the proof of Proposition C.14.

\section{References}

[1] Beck. J., Braid group action and quantum affıne algebras, Comm. Math. Phys., 165 (1994) 555-568.

[2] Chari, V. and Pressley, A., A Guide to Quantum Gronts, Cambridge University Press, 1994.

[3] Q Quantum affine algebras and their representations, Canad. Math. Soc. Conf. Proc., 16 (1995). 59-78.

[4] _ Twisted quantum affine algebras, q-alg/9611002.

[5] Date, E. and Okado, M., Calculation of excitation spectra of the spin model related with the vector represention of the quantized affıne algebra of type $A_{n}^{(1)}$. Interia. J Modcrn Phys. A, 9 (1994), 399-417.

[6] Drinfeld, V. G., Quantum grouts. Proc. ICM-86 (Berkeley), AMS, 1. 798-820.

[7] A new realization of Yangıans and the quantum affine algebras, Soviet. Math. Dokl., 36 (1988), 212-216.

[8] Frenkel, I. and Reshetıkhin. N., Quantum affıne algebras and holonomic difference equations. Comm. Math. Phys., 146 (1992), 1-60.

[9] Gandenberger, G. M. and MacKay. N. J., Exact S-matrices for $d_{n+1}^{(2)}$ affine Toda solitons and their bounded states. Nucleur Plys. B457 (1995), 240-272.

[10] Jimbo, M., A $q$-difference analogue of $U(\mathrm{~g})$ and the Yang Baxter equation, Lett. Math. Phys., 10 (1985), 63-69.

[11] Jimbo, M. and Miwa. T., Algebraic Analysis of Solvable Lattrce Models, CBMS Regronal Conf. series in Math., 85, AMS. 1995.

[12] Kashiwara, M., On crystal bases of the $q$-analogue of universal enveloping algebras, Duke Math. J., 63 (1991), 465-516.

[13] . Global crystal bases of quantum groups, Duke Math. J., 69 (1993). 455-485.

[14] _. Crystal bases of modified quantized enveloping algebra, Duke Math. J., 73 (1994), 383-413.

[15] Kang, S.-J., Kashıwara. M., Mısra. K., Miwa. T., Nakashıma, T.,and Nakayashıki. A.. Affine crystal and Vertex models, Internat. J. Modem Phys. A 7. Suppl. 1A (1992), 449-484.

[16] ___. Perfect crystals of quantum affine Lie algebra. Durk Math. J., 68 (1992) 499-607.

[17] Kashiwara. M. and Nakashima. T., Crystal graphs for representations of the $q$-analogue of classical Lie algebras. J. Algebra. 165 (1994). 295-345.

[18] Tanisaki, T., Killnng forms, Harish-Chandra isomorphisms, and universal R-matrices for quantum algcbras, Infinite Analysis, Proceedings of the RIMS Project 1991, Part B, Adv. Ser. Math. Phys. 16, World Scientıfic, (1992) 941-962. 
